Article

\title{
Synthesis and Antifungal Activity of Benzamidine Derivatives Carrying 1,2,3-Triazole Moieties
}

\section{Guangyou Chen, Yiwan Zhou, Chonglin Cai, Jia Lu and Xing Zhang *}

Research \& Development Center of Biorational Pesticide, Key Laboratory of Plant Protection Resources and Pest Management of Ministry of Education, Northwest A\&F University, Yangling 712100 , China

* Author to whom correspondence should be addressed; E-Mail: zhxing1952@126.com; Tel./Fax: +8-629-870-921-22.

Received: 18 March 2014; in revised form: 20 April 2014 / Accepted: 25 April 2014 /

Published: 2 May 2014

\begin{abstract}
Eighteen novel benzamidine derivatives containing 1,2,3-triazole moieties were synthesized. The in vitro and in vivo fungicidal acitivities of the title compounds and the arylamidine intermediates against Colletotrichum lagenarium and Botrytis cinerea were tested. The synthesized benzamidines exhibited weak antifungal activities in vitro against the tested fungi, but some of the compounds showed excellent activities in vivo to the same strains. Among the compounds tested, 9 b showed $79 \%$ efficacy in vivo against C. lagenarium at a concentration of $200 \mu \mathrm{g} / \mathrm{mL}$, and the efficacy of compound $\mathbf{1 6 d}(90 \%)$ toward the same strain was even superior than that of the commercial fungicide carbendazim $(85 \%)$.
\end{abstract}

Keywords: amidine; 1,2,3-triazole; antifungal activity; synthesis

\section{Introduction}

The majority of agricultural phytopathogens, especially Botrytis cinerea, the causal agent of the common disease gray mold on vegetable and fruit crops, have developed resistance to the most commonly used fungicides all over the world [1]. Application of synthetic fungicides is still the most efficient tool to control crop diseases. This situation urged us to find new compounds having agricultural antifungal activities. Amidine derivatives exhibit several significant bioactivities, such as antitumor [2], trypanocidal [3-5], antiprotozoan [6,7], anti-HIV [8], diuretic, anti-inflammatory, 
analgesic [9], antivirus, fungicidal and bactericidal [10-13] activities. Amidines can also be used in the synthesis of metallo-organic compounds [14]. Aromatic diamidines has been explored as agents against a widespread range of microorganisms [15]. In our previous works, aromatic diamidines were found to have excellent antifungal activities in agriculture, especially for the treatment of gray mold on tomatoes $[16,17]$. It was reported that good bioacitivities could be obtained by linking the arylamidino group with heteorocycle moieties [18-20].

1,2,3-Triazoles are very important heterocycles showing excellent bioactivities in pharmaceutical and agrochemical applications. Compounds containing 1,2,3-triazoles have been reported for their antifungal [21-23], antibacterial [24-27], anti-HIV [28], insecticidal [29], herbicidal [30], antiviral [31], antitumor [32], tuberculosis inhibitory [33], antiprotozoal [34], antimalarial [35], anticancer [36], and larvicidal [37] activities. The fungicidal activity of the commercial fungicide fluconazole can be improved by introducing a 1,2,3-triazole moiety into the molecule [38,39]. The presence of the 1,2,3-triazole moiety in oxazolidinone compounds can increase the protein binding ability and subsequently improve the antibacterial activities [40]. The compounds formed by linking a 1,2,3-triazole ring and an indole nucleus together possessed much higher antimicrobial activity compared with the precursors [41]. Another advantage of 1,2,3-triazoles is that they can be prepared conveniently by Huisgen's cycloaddition reaction between azides and terminal alkynes without complicated purification.

In an attempt to find more potent antifungal molecules, a series of new compounds were designed by linking aromatic amidines and 1,2,3-triazoles. In addition, their in vitro and in vivo biological activity toward the strains $C$. lagenarium and $B$. cinerea were studied in this paper.

\section{Results and Discussion}

\subsection{Chemistry}

Target molecules 4a-e were synthesized according to Scheme 1. 4-(Azidomethyl)benzonitrile (2) was obtained by adding aqueous sodium azide to 4-cyanobenzyl bromide in DMF [42], the reaction took place quantitatively and the product can be separated by simple extraction. The potentially explosive benzyl azide 2 should be handled carefully and the precipitation should be avoided by first adding toluene to the reaction mixture in the end of the reaction. After being dried, $\mathbf{2}$ can be used as a solution without evaporation of the solvent. Then compound $\mathbf{2}$ was reacted with propargyl alcohol under standard "click chemistry" [43] conditions using a copper sulfate/sodium ascorbate system as the catalyst. The copper sulfate and sodium ascorbate were dissolved in water before being added to the reaction mixture to ensure a good dispersion of the resultant cuprous salt. The temperature is crucial in this step. Little 2 had been converted and the mixture stayed clear when the reaction mixture being stirred for $48 \mathrm{~h}$ at $40{ }^{\circ} \mathrm{C}$. However, plenty of crystals were formed in the flask after being stirred for $6 \mathrm{~h}$ at $55^{\circ} \mathrm{C}$ when TLC indicated that no 2 remained. The target compound was obtained in high yield by recrystallization. 4-((4-(Hydroxymethyl)-1H-1,2,3-triazol-1-yl) methyl)benzonitrile (3) was converted into 4 by a Pinner reaction [44]. Anhydrous conditions were important in the synthesis of the Pinner salt. Compound 3 was dried under $110^{\circ} \mathrm{C}$ before use, and absolute ethanol was adopted as both solvent and reactant. The moisture-sensitive Pinner salt was handled quickly when being filtered and recrystallized in order to reduce byproducts. The Pinner salt was dissolved in absolute ethanol and kept 
under anhydrous conditions before use to avoid the absorption of moisture. Compounds $\mathbf{4 a - e}$ were obtained by the ammonization of the Pinner salt with corresponding amines.

Scheme 1. Synthesis of compounds 4 and 9.

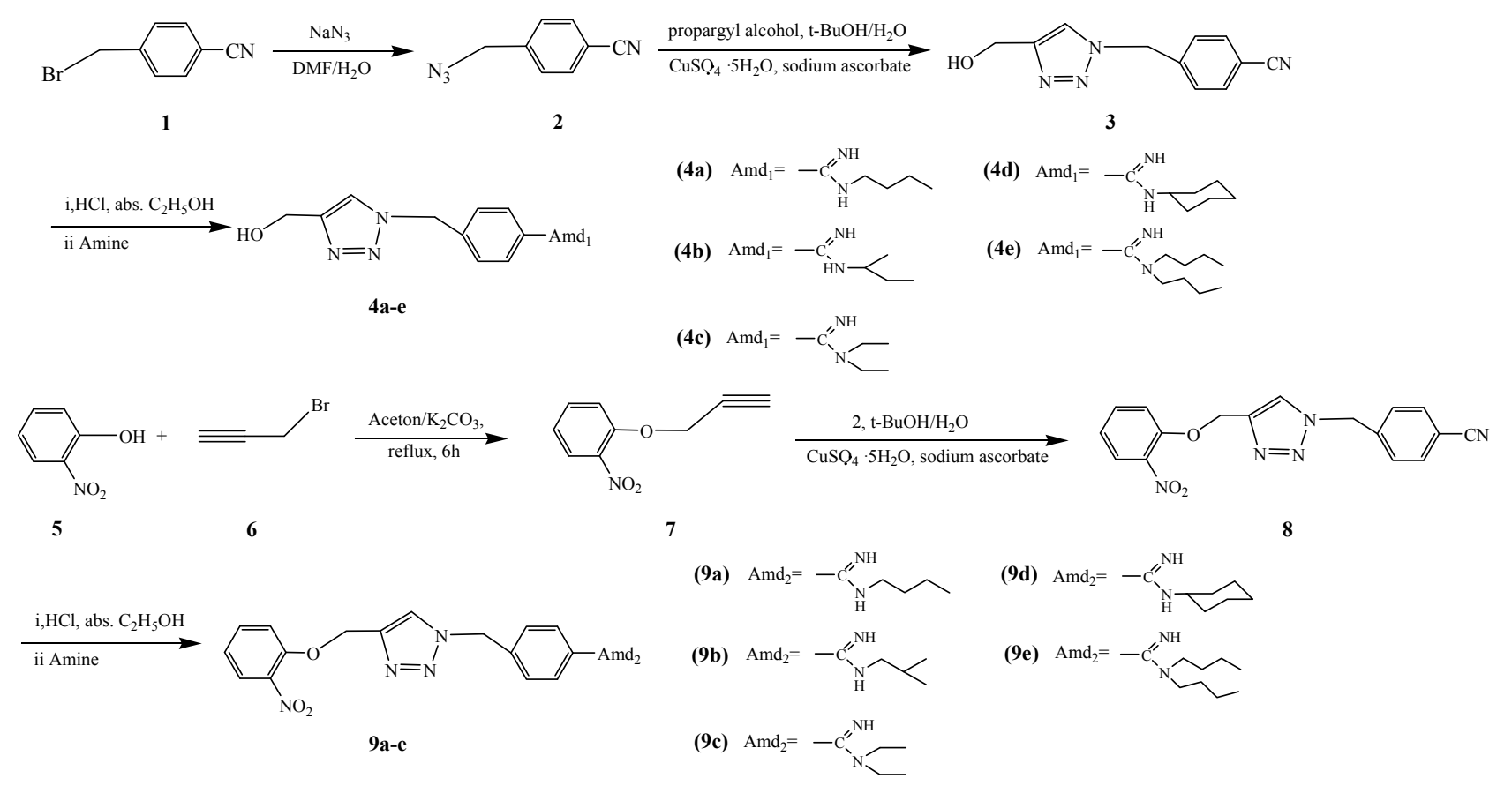

Compounds 9a-e were obtained according to the procedure described in Scheme 1. 4-((4-)((2Nitrophenoxy)methyl)-1H-1,2,3-triazol-1-yl)methyl)-benzonitrile (8) was obtained by reacting 1-nitro-2-(prop-2-ynyloxy) benzene (7) with 2 under "click chemistry" conditions. Compound 8 was converted into the corresponding amidines 9 following the same procedure used for 4.

When we applied the method used for the preparation of 4 and 9 in synthesizing 16 and 17, the target products could not be obtained. Compounds 18 and 19 were obtained in high yields by reacting 10 with propargyl alcohol or 12, respectively. However, when treated with hydrogen chloride in anhydrous ethanol, the cyano group on $\mathbf{1 8}$ or $\mathbf{1 9}$ could not be converted into the corresponding Pinner salt. We surmised that the unreactiveness of the cyano group on the 3-position of the benzene ring may be attributed to the electron donating aminomethyl group at the meta position [45], which decreased the electron density at the position the cyano group is linked to, and made it very difficult for the $\mathrm{HCl}$-catalyzed Pinner reaction [46] to occur. The synthesis strategy was therefore adjusted as shown in Scheme 2. The 3-(azidomethyl)benzonitrile was inverted into the corresponding amidines by a Pinner reaction [42] before the 3-(azidomethyl)-N-benzamidines 15a-e were connected with 1-nitro-4-(prop-2-ynyloxy)benzene (12) or propargyl alcohol using the "click chemistry" method to obtain the target compounds.

The chemical structures of the target compounds (Table 1) were confirmed by their spectral data $\left({ }^{1} \mathrm{H}-\mathrm{NMR},{ }^{13} \mathrm{C}-\mathrm{NMR}\right.$, ESI-MS, and elementary analysis). The ${ }^{1} \mathrm{H}-\mathrm{NMR}$ spectra of compounds 4, 9, 16 and 17 showed a single $\mathrm{NH}$ signal from the triazole group at 7.95-8.25 ppm [47,48] and the signal of $\mathrm{CH}$ (or $\mathrm{CH}_{2}$ ) attached to the amidines at 2.97-3.89 ppm [49]. The ${ }^{13} \mathrm{C}-\mathrm{NMR}$ spectra of the compounds 4, 9, 15, 16 and 17 showed the signal of $\mathrm{C}=\mathrm{N}$ in the amidino group at 162-166 ppm [50]. Based on the abovementioned data, it can be concluded that the structures of the amidines were identified correctly. 
Scheme 2. Synthesis of compounds 16 and 17.

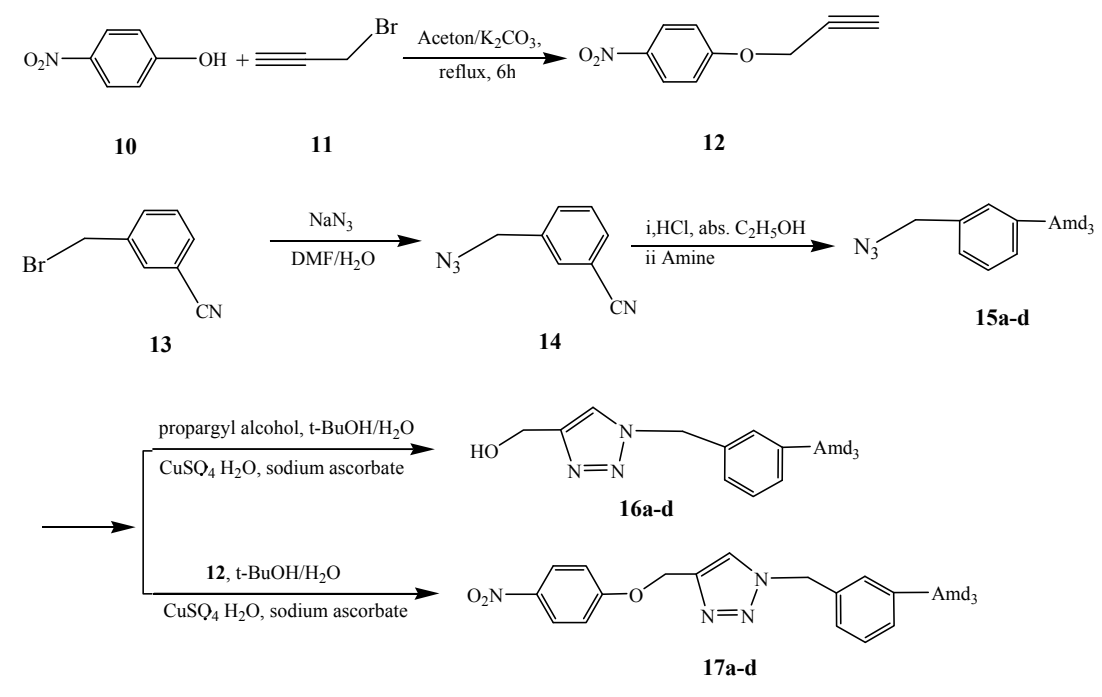

$10 \underset{\text { propargyl alcohol, } \mathrm{t}-\mathrm{BuOH} / \mathrm{H}_{2} \mathrm{O}}{\mathrm{CuSO_{4 } \mathrm { H } _ { 2 } \mathrm { O } \text { , sodium ascorbate }} \rightarrow \mathrm{HO}} \rightarrow$

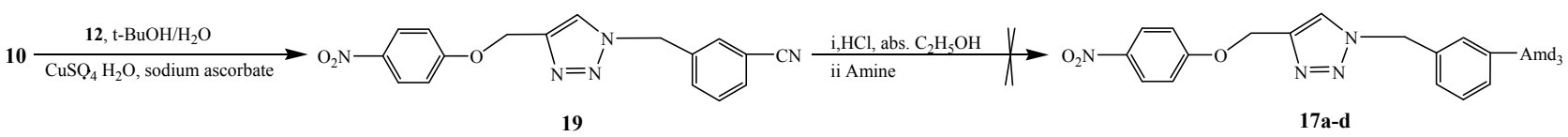

19

17a-d

$\mathrm{Amd}_{3}=\mathrm{C}_{\mathrm{H}}^{\mathrm{N}} \mathrm{N}_{\mathrm{H}}^{\mathrm{NH}}(15 \mathbf{a}, 16 \mathbf{a}, 17 \mathbf{a}) \quad(15 \mathbf{b}, 16 \mathbf{b}, 17 \mathbf{b})$

$-\mathrm{C}_{\mathrm{N}}^{\mathrm{NH}} \sim(15 \mathrm{~d}, 16 \mathrm{~d}, 17 \mathrm{~d})$

Table 1. Inhibition activity (\%) of the amidines on phytopathogenic fungi ${ }^{\text {a }}$.

\begin{tabular}{ccccc}
\hline \multirow{2}{*}{ Compounds s } & \multicolumn{4}{c}{ Inhibition rate $(\%)$} \\
\cline { 2 - 5 } & \multicolumn{2}{c}{ in vitro $(\mathbf{2 0 0} \boldsymbol{\mu g} / \mathbf{m L})$} & in vivo $(\mathbf{3 0 0} \boldsymbol{\mu g} / \mathbf{m L})$ \\
\cline { 2 - 5 } C. lagenarium & B. cinerea & C. lagenarium & B. cinerea \\
\hline 4a & 26 & 23 & 49 & 19 \\
4c & 19 & 14 & 30 & 60 \\
4d & 17 & 25 & 60 & 23 \\
4e & 8 & 0 & 0 & 16 \\
9a & 10 & 12 & 48 & 56 \\
9b & 32 & 22 & 57 & 24 \\
9c & 31 & 30 & 79 & 49 \\
9d & 61 & 17 & 4 & 13 \\
9e & 29 & 44 & 17 & 35 \\
15a & 35 & 14 & 45 & 14 \\
15b & 12 & 16 & 31 & 43 \\
15c & 25 & 19 & 61 & 68 \\
15d & 12 & 24 & 0 & 48 \\
\hline
\end{tabular}


Table 1. Cont.

\begin{tabular}{ccccc}
\hline \multirow{2}{*}{ Compounds } & \multicolumn{4}{c}{ Inhibition rate (\%) } \\
\cline { 2 - 5 } & \multicolumn{2}{c}{ in vitro $(\mathbf{2 0 0} \boldsymbol{\mu g} / \mathbf{m L})$} & in vitro $(\mathbf{2 0 0} \boldsymbol{\mu g} / \mathbf{m L})$ \\
\cline { 2 - 5 } & C. lagenarium & B. cinerea & C. lagenarium & B. cinerea \\
\hline $\mathbf{1 6 a}$ & 25 & 15 & 19 & 11 \\
$\mathbf{1 6 b}$ & 16 & 8 & 56 & 32 \\
$\mathbf{1 6 c}$ & 10 & 5 & 52 & 47 \\
$\mathbf{1 6 d}$ & 26 & 13 & 90 & 7 \\
$\mathbf{1 7 a}$ & 65 & 36 & 52 & 74 \\
$\mathbf{1 7 b}$ & 21 & 19 & 27 & 0 \\
$\mathbf{1 7 c}$ & 32 & 12 & 45 & 45 \\
$\mathbf{1 7 d}$ & 10 & 17 & 53 & 31 \\
Carbendazim & 100 & 100 & 85 & 61 \\
\hline \multicolumn{2}{c}{${ }^{\mathrm{a}}$} & The data are the average of three duplicated results.
\end{tabular}

\subsection{Antifungal Activity Bioassay}

The in vitro and in vivo fungicidal activities of the amidines $4 \mathbf{a}-\mathbf{e}, 9 \mathbf{a}-\mathbf{e}, 16 \mathbf{a}-\mathbf{d}, 17 \mathbf{a}-\mathbf{d}$ and $15 \mathbf{a}-\mathbf{d}$ are listed in Table 1. Some of the compounds showed valuable in vitro inhibitory activity against C. lagenarium and B. cinerea at a concentration of $200 \mu \mathrm{g} / \mathrm{mL}$. The test results showed that some of the title compounds and the intermediates 3-(azidomethyl)-N-benzamidine 15a-d possessed fairly potent antifungal activities in vivo to both $C$. lagenarium and $B$. cinerea at the concentration of $300 \mu \mathrm{g} / \mathrm{mL}$. The antifungal activity of those compounds was compared with that of the the commercial fungicide carbendazim at the same concentration. Compounds 9c and 17a exhibited a significant in vitro inhibition effect against $C$. lagenarium, but the inhibition activities (control efficacy of $61 \%$ and $65 \%$ ) were less than those of the fungicide carbendazim (100\%). The analysis above indicated that the variation of the substituent groups on the 1,2,3-triazole ring had a great effect on the antifungal activity against $C$. lagenarium. The activities of most compounds in the 9 and 17 series having nitrophenoxyl groups on the 1,2,3-triazole ring were higher than those of the 4 and $\mathbf{1 6}$ series with hydroxymethyl ones. For example, the activities of compounds $\mathbf{4 c}$ and $\mathbf{4 d}$ were $17 \%$ and $9 \%$, while the activities of $9 \mathbf{c}$ and $9 d$ were increased to $61 \%$ and $29 \%$. The cyclization of azido groups with propargyl alcohol had no obvious effect on the antifungal activities. However, when propargyl alcohol was replaced with 1-nitro-4-(prop-2-ynyloxy)benzene, the activities were increased significantly. The substituents on the amidino group and the position of the amidino group on the phenyl ring also had significant effects on the antifungal activities, but no rules could be extracted from the activity data. The in vitro activity of the compounds to B. cinerea obeyed the same rule, however, it was not as obvious as the one drawn from the C. lagenarium data. Some of the compounds exhibited significant activities in vivo toward $C$. lagenarium, in which the compounds 4c, 9a, 9b, 16d and 15b had control efficacy rates of 60\%, 57\%, 79\% and 61\%, respectively. It was noteworthy that compound $\mathbf{1 6 d}$ was found to be even more effective than the fungicide carbendazim (control efficacy of $85 \%$ ). For $B$. cinerea, many compounds showed excellent in vivo antifungal activities. Compounds $\mathbf{4 b}, \mathbf{4 e}$, and $\mathbf{1 7} \mathbf{a}$ and $\mathbf{1 5 b}$, whose efficacy rates were $60 \%$, $56 \%, 75 \%$, and $68 \%$, respectively, were found to be much more effective as compared to the 
fungicide carbendazim SC (54\%) against $B$. cinerea. The different substituents at the 4-position of the triazole ring and amidino group had no obvious relationship with the fungicidal activity against B. cinerea. Most of the compounds exhibited more effective activities in vivo, especially for $B$. cinerea, than ones in vitro when compared with the commercial fungicide. The aryl amidine moieties played a very crucial role in the antifungal activities.

\section{Experimental}

\subsection{General Information}

Melting points were measured on an X-4 melting point apparatus (Beijing Tech. Instrument Co., Beijing, China), and were not corrected. Mass spectra were obtained on a Thermo-Finnigan LCQ-Advantage (ESI) instrument (Thermo Electron Corporation, Waltham, MA, USA); ${ }^{1} \mathrm{H}-\mathrm{NMR}$ and ${ }^{13} \mathrm{C}$-NMR spectra were obtained on Bruker $400 \mathrm{MHz}$ or $500 \mathrm{MHz}$ spectrometers (Bruker Corporation, Billerica, MA, USA) in $\mathrm{CDCl}_{3}, \mathrm{CDOD}_{3}$, or $\left(\mathrm{CD}_{3}\right)_{2} \mathrm{SO}$ solution; elemental analysis was performed on an Elemental Vario-III CHN analyzer (Elementar Analysensysteme GmbH, Hanau, Germany). Reagents and solvents were all chemically or analytically pure without further purification if not mentioned. Anhydrous solvents were purified by standard techniques before use. Anhydrous $\mathrm{HCl}$ (gas) was prepared by the addition of concentrated hydrochloric acid dropwise to concentrated sulfuric acid and dried by the latter. Reaction progress was monitored by TLC on silica gel F254 plates prepared in the laboratory, and was visualized under $\mathrm{UV}_{254}$ and iodine.

\subsection{Synthesis of 4-(Azidomethyl)benzonitrile (2) [42]}

4-Cyanobenzyl bromide (1, 45.10 g, $230 \mathrm{mmol})$ was dissolved in DMF (200 $\mathrm{mL})$ in a flask equipped with a thermometer, and a solution of $\mathrm{NaN}_{3}(19.80 \mathrm{~g}, 300 \mathrm{mmol})$ in distilled water $(100 \mathrm{~mL})$ was added to it in batches under ice bath cooling with stirring, keeping the reaction mixture under $15^{\circ} \mathrm{C}$. The reaction mixture was stirred for 2 more $\mathrm{h}$ at ambient temperature after the addition of the sodium azide until TLC indicated no 1 remained. The mixture was transferred to a conical flask and diluted with toluene $(200 \mathrm{~mL})$ and distilled water $(600 \mathrm{~mL})$. The mixture was separated in a separating funnel and the aqueous phase was extracted with toluene $(2 \times 70 \mathrm{~mL})$. The organic extracts were combined and washed with brine $(70 \mathrm{~mL})$ and water $(2 \times 70 \mathrm{~mL})$ and dried by $\mathrm{MgSO}_{4}$ overnight. A solution of 2 in toluene $(310 \mathrm{~mL})$ was obtained after filtration. After the solvent was evaporated under reduced pressure, 2 was obtained as light yellow oil (35.69 g, 98.2\%), ${ }^{1} \mathrm{H}-\mathrm{NMR}\left(500 \mathrm{MHz}, \mathrm{CDCl}_{3}\right) \delta$ : $7.61\left(\mathrm{~d}, J=8.2 \mathrm{~Hz}, 2 \mathrm{H}\right.$, benzene-H), $7.41\left(\mathrm{~d}, J=7.4 \mathrm{~Hz}, 2 \mathrm{H}\right.$, benzene-H), $4.42\left(\mathrm{~s}, 2 \mathrm{H}, \mathrm{CH}_{2}\right) .{ }^{13} \mathrm{C}-\mathrm{NMR}$ $\left(125 \mathrm{MHz}, \mathrm{CDCl}_{3}\right) \delta: 140.97,132.82,132.55,132.55,129.07,128.56,128.56,128.27,125.35,118.55$, $111.92,77.80,77.55,77.29,53.90,53.90,21.37$.

\subsection{Synthesis of 4-((4-(Hydroxymethyl)-1H-1,2,3-triazol-1-yl)methyl) Benzonitrile (3)}

Compound $2(35.1 \mathrm{~g})$ was dissolved in a mixture of $t$-BuOH $(300 \mathrm{~mL})$ and distilled water $(100 \mathrm{~mL})$, then propargyl alcohol $(12.3 \mathrm{~g}, 230 \mathrm{mmol})$ was added. The reaction mixture was stirred in a water bath at $60{ }^{\circ} \mathrm{C}$ for $48 \mathrm{~h}$ after the addition of sodium ascorbate $(306 \mathrm{mg}$, in $3 \mathrm{~mL}$ of water) and cupric sulfate $\left(\mathrm{CuSO}_{4} \cdot 5 \mathrm{H}_{2} \mathrm{O}\right)(105 \mathrm{mg}$, in $2 \mathrm{~mL}$ of water $)$. The clear reaction mixture turned light yellow. 
Compound 3 (46.65 g, $128 \mathrm{mmol}, 81 \%$ ) was obtained as white needle-like crystals after filtration and dried under $110{ }^{\circ} \mathrm{C}$ for $7 \mathrm{~h}$ when most of the solvent was evaporated under vacuum; m.p. 136-137 ${ }^{\circ} \mathrm{C} .{ }^{1} \mathrm{H}-\mathrm{NMR}(400 \mathrm{MHz}, \mathrm{DMSO}) \delta: 8.09$ (s, 1H, triazole-H), $7.86(\mathrm{~d}, J=8.3 \mathrm{~Hz}, 2 \mathrm{H}$, benzene-H), 7.46 (d, $J=8.3 \mathrm{~Hz}, 2 \mathrm{H}$, benzene-H), $5.70\left(\mathrm{~s}, 2 \mathrm{H}, \mathrm{CH}_{2}\right), 5.22(\mathrm{t}, J=5.6 \mathrm{~Hz}, 1 \mathrm{H}, \mathrm{OH})$, 4.53 (d, $\left.J=5.4 \mathrm{~Hz}, 2 \mathrm{H}, \mathrm{CH}_{2}\right) .{ }^{13} \mathrm{C}-\mathrm{NMR}$ (100 MHz, DMSO) $\delta: 148.97,142.19,133.19,129.10$, $123.73,119.04,111.34,55.49,52.53$.

\subsection{General Procedure for the Synthesis of 4-((4-(Hydroxymethyl)-1H-1,2,3-triazol-1-yl) Methyl)- benzamidines $\mathbf{4 a}-\mathbf{e}$}

A suspension of $3(3.0 \mathrm{~g}, 14.0 \mathrm{mmol})$ in absolute ethanol $(50 \mathrm{~mL})$ was cooled to below $5{ }^{\circ} \mathrm{C}$ and saturated with dry $\mathrm{HCl}$ gas. Then the mixture was sealed and stirred at ambient temperature for $48 \mathrm{~h}$. White crystalline imino ester was obtained after evaporation of the solvent under anhydrous conditions and washing with dry ether $(3 \times 15 \mathrm{~mL})$. The resulting imino ester was dissolved in absolute methanol $(20 \mathrm{~mL})$. An exothermic reaction took place and the mixture became clear when it was treated with the corresponding amines $(60 \mathrm{mmol})$. Keeping the reaction mixture stirring overnight in sealed flasks resulted in the formation of the corresponding amidines. After evaporation of the solvent in anhydrous conditions, the residue was dissolved in a mixture of ethanol $(5 \mathrm{~mL})$ and $2 \mathrm{~N}$ aqueous $\mathrm{NaOH}(5 \mathrm{~mL})$. The target product was precipitated by the addition of ether $(40 \mathrm{~mL})$ to the organic phase and then purified by column chromatography on silica gel $\left(\mathrm{CH}_{2} \mathrm{Cl}_{2} / \mathrm{MeOH}=6: 1\right)$. Compounds $4 \mathbf{a}-\mathbf{d}$ were prepared by the same method.

N-Butyl-4-((4-(hydroxymethyl)-1H-1,2,3-triazol-1-yl)methyl)benzamidine (4a): Yield, 42.1\%; white crystals; m.p. $111-112{ }^{\circ} \mathrm{C} .{ }^{1} \mathrm{H}-\mathrm{NMR}(400 \mathrm{MHz}, \mathrm{MeOD}) \delta: 8.05$ (d, $J=12.1 \mathrm{~Hz}, 1 \mathrm{H}$, triazole-H), 7.75 (d, $J=8.2 \mathrm{~Hz}, 2 \mathrm{H}$, benzene-H), $7.53\left(\mathrm{~d}, J=8.2 \mathrm{~Hz}, 2 \mathrm{H}\right.$, benzene-H), $5.75\left(\mathrm{~s}, 2 \mathrm{H}, \mathrm{CH}_{2}\right), 5.69$ (d, $J=6.0 \mathrm{~Hz}$, $\left.2 \mathrm{H}, \mathrm{CH}_{2}\right), 3.42\left(\mathrm{t}, J=7.3 \mathrm{~Hz}, 2 \mathrm{H}, \mathrm{N}-\mathrm{CH}_{2}\right), 1.83-1.73\left(\mathrm{~m}, 2 \mathrm{H}, \mathrm{CH}_{2}\right), 1.05\left(\mathrm{t}, J=7.4 \mathrm{~Hz}, 3 \mathrm{H}, \mathrm{CH}_{3}\right)$. ${ }^{13} \mathrm{C}-\mathrm{NMR}(100 \mathrm{MHz}, \mathrm{MeOD}) \delta$ : 166.56, 141.31, 129.99, 129.68, 129.16, 128.23, 127.76, 60.92, 54.98, 44.61, 20.73, 10.25. MS (ESI $\left.{ }^{+}\right) \mathrm{m} / z: 288[\mathrm{M}+\mathrm{H}]^{+}$. Anal. Calc. for $\mathrm{C}_{15} \mathrm{H}_{21} \mathrm{~N}_{5} \mathrm{O} \cdot \mathrm{HCl}(323.15): \mathrm{C}, 55.64 ; \mathrm{H}$, $6.85 ; \mathrm{N}, 21.63$; found: C, 55.11; H, 6.62; N, 21.56.

N-Sec-butyl-4-((4-(hydroxymethyl)-1H-1,2,3-triazol-1-yl)methyl)benzamidine (4b): Yield， 47.4\%; white crystals; m.p. $118-120{ }^{\circ} \mathrm{C} .{ }^{1} \mathrm{H}-\mathrm{NMR}(400 \mathrm{MHz}, \mathrm{MeOD}) \delta: 8.02(\mathrm{~s}, 1 \mathrm{H}$, triazole-H), $7.71(\mathrm{~d}$, $J=8.2 \mathrm{~Hz}, 2 \mathrm{H}), 7.52\left(\mathrm{~d}, J=8.2 \mathrm{~Hz}, 2 \mathrm{H}\right.$, benzene-H), $5.72\left(\mathrm{~s}, 2 \mathrm{H}, \mathrm{CH}_{2}\right), 4.65\left(\mathrm{~s}, 2 \mathrm{H}, \mathrm{CH}_{2}\right), 3.81(\mathrm{dd}$, $J=13.2,6.6 \mathrm{~Hz}, 1 \mathrm{H}, \mathrm{CH}), 3.28\left(\mathrm{~m}, 2 \mathrm{H}, \mathrm{N}-\mathrm{CH}_{2}\right), 1.62-1.48\left(\mathrm{~m}, 3 \mathrm{H}, \mathrm{CH}_{3}\right), 1.31(\mathrm{~d}, J=6.5 \mathrm{~Hz}, 3 \mathrm{H}$, $\mathrm{CH}_{3}$ ). MS $\left(\mathrm{ESI}^{+}\right) \mathrm{m} / z: 288[\mathrm{M}+\mathrm{H}]^{+}$. Anal. Calc. for $\mathrm{C}_{15} \mathrm{H}_{21} \mathrm{~N}_{5} \mathrm{O} \cdot \mathrm{HCl}(323.15): \mathrm{C}, 55.64 ; \mathrm{H}, 6.85 ; \mathrm{N}$, 21.63; found: C, 55.23; H, 6.71; N, 21.75 .

N,N-Diethyl-4-((4-(hydroxymethyl)-1H-1,2,3-triazol-1-yl)methyl)benzamidine (4c): Yield, 42.1\%; yellow crystals; m.p. $>300{ }^{\circ} \mathrm{C} .{ }^{1} \mathrm{H}-\mathrm{NMR}(500 \mathrm{MHz}, \mathrm{DMSO}) \delta: 8.13(\mathrm{~s}, 1 \mathrm{H}$, triazole-H), $8.06(\mathrm{~d}, J=8.3 \mathrm{~Hz}, 2 \mathrm{H}$, benzene-H), $7.46\left(\mathrm{~d}, J=8.3 \mathrm{~Hz}, 2 \mathrm{H}\right.$, benzene-H), $5.71\left(\mathrm{~s}, 2 \mathrm{H}, \mathrm{CH}_{2}\right), 4.51$ (d, $\left.J=5.1 \mathrm{~Hz}, 2 \mathrm{H}, \mathrm{CH}_{2}\right), 3.89$ (s, $\left.4 \mathrm{H}, \mathrm{N}-\mathrm{CH}_{2}\right) .{ }^{13} \mathrm{C}-\mathrm{NMR}(125 \mathrm{MHz}, \mathrm{DMSO}) \delta$ : 164.49, 148.93, 142.23, 129.28, 128.63, 124.19, 123.73 , 55.46, 52.57, 45.94. MS (ESI $\left.{ }^{\dagger}\right) m / z: 288[\mathrm{M}+\mathrm{H}]^{+}$. Anal. Calc. for $\mathrm{C}_{15} \mathrm{H}_{21} \mathrm{~N}_{5} \mathrm{O} \cdot \mathrm{HCl}$ (323.15): C, 55.64; $\mathrm{H}$, $6.85 ; \mathrm{N}, 21.63$; found: C, 55.37; H, 6.65; N, 21.73. 
N-Cyclohexyl-4-((4-(hydroxymethyl)-1H-1,2,3-triazol-1-yl)methyl)benzamidine (4d): Yield, 42.1\%; white crystals; m.p. 171-173 ${ }^{\circ} \mathrm{C} .{ }^{1} \mathrm{H}-\mathrm{NMR}(400 \mathrm{MHz}, \mathrm{MeOD}) \delta: 8.21$ (s, 1H, triazole-H), 7.79 (d, $J=8.1,1.5 \mathrm{~Hz}, 2 \mathrm{H}$, benzene-H), $7.73\left(\mathrm{~d}, J=8.3 \mathrm{~Hz}, 2 \mathrm{H}\right.$, benzene-H), $5.77\left(\mathrm{~s}, 2 \mathrm{H}, \mathrm{CH}_{2}\right), 5.35(\mathrm{~s}, 2 \mathrm{H}$, $\left.\mathrm{CH}_{2}\right), 3.82$ (d, $\left.J=6.7 \mathrm{~Hz}, 1 \mathrm{H}, \mathrm{N}-\mathrm{CH}\right), 1.79-1.65\left(\mathrm{~m}, 4 \mathrm{H}, \mathrm{CH}_{2}\right), 1.65-1.52\left(\mathrm{~m}, 2 \mathrm{H}, \mathrm{CH}_{2}\right), 1.34$ (d, $\left.J=6.5 \mathrm{~Hz}, 3 \mathrm{H}, \mathrm{CH}_{2}\right), 1.04\left(\mathrm{t}, J=7.3 \mathrm{~Hz}, 3 \mathrm{H}, \mathrm{CH}_{2}\right) . \mathrm{MS}\left(\mathrm{ESI}^{+}\right) \mathrm{m} / z: 314[\mathrm{M}+\mathrm{H}]^{+}$. Anal. Calc. for $\mathrm{C}_{17} \mathrm{H}_{23} \mathrm{~N}_{5} \mathrm{O} \cdot \mathrm{HCl}$ (349.17): C, 58.36; H, 6.91; N, 20.02; found: C, 57.97; H, 6.85; N, 20.34.

N,N-Dibutyl-4-((4-(hydroxymethyl)-1H-1,2,3-triazol-1-yl)methyl)benzamidine (4e): Yield, 42.1\%; white crystals; m.p. $111-112{ }^{\circ} \mathrm{C} .{ }^{1} \mathrm{H}-\mathrm{NMR}(400 \mathrm{MHz}, \mathrm{MeOD}) \delta: 7.99$ (s, 1H, triazole-H), 7.87-7.82 (m, 2H, benzene-H), 7.47-7.43 (m, 2H, benzene-H), $5.69\left(\mathrm{~s}, 2 \mathrm{H}, \mathrm{CH}_{2}\right), 5.65\left(\mathrm{~s}, 2 \mathrm{H}, \mathrm{CH}_{2}\right), 2.81-2.74$ $\left(\mathrm{m}, 4 \mathrm{H}, \mathrm{N}-\mathrm{CH}_{2}\right), 1.80\left(\mathrm{~d}, J=7.5 \mathrm{~Hz}, 4 \mathrm{H}, \mathrm{CH}_{2}\right), 1.24\left(\mathrm{~m}, 4 \mathrm{H}, \mathrm{CH}_{2}\right), 0.80$ (t, $\left.J=7.3 \mathrm{~Hz}, 6 \mathrm{H}, \mathrm{CH}_{3}\right) . \mathrm{MS}$ $\left(\mathrm{ESI}^{+}\right) \mathrm{m} / z: 344[\mathrm{M}+\mathrm{H}]^{+}$. Anal. Calc. for $\mathrm{C}_{19} \mathrm{H}_{29} \mathrm{~N}_{5} \mathrm{O} \cdot \mathrm{HCl}$ (379.21): C, 60.06; H, 7.96; N, 18.43; found: C, 59.78; H, 7.81; N, 18.71.

\subsection{Synthesis of 1-Nitro-2-(prop-2-ynyloxy) Benzene (7)}

2-Nitrophenol $(5,9.10 \mathrm{~g}, 65 \mathrm{mmol})$ and $\mathrm{K}_{2} \mathrm{CO}_{3}(9.0 \mathrm{~g}, 65 \mathrm{mmol})$ were dissolved in acetone $(60 \mathrm{~mL})$ in a three necked flask equipped with a thermometer. Then a solution of propargyl bromide (16.03 $\mathrm{g}$, $300 \mathrm{mmol})$ in acetone $(30 \mathrm{~mL})$ was added dropwise at ambient temperature in about 15 min while stirring. Then the reaction mixture was heated to reflux for $3 \mathrm{~h}$ after being stirred for 30 min when TLC (petroleum ether/ethyl acetate $=6: 1$ ) indicated no 5 remained. The mixture was allowed to cool to ambient temperature and the resulting white solid was recrystallized in ethanol, to afford yellowish needle-shaped crystals of 7 (8.11 g, 70\%); m.p. 115-116 ${ }^{\circ} \mathrm{C} .{ }^{1} \mathrm{H}-\mathrm{NMR}\left(400 \mathrm{MHz}, \mathrm{CDCl}_{3}\right) \delta: 7.85$ (dd, $J=8.1,1 \mathrm{H}$, benzene-H), 7.64-7.51 (m, 1H, benzene-H), 7.27 (t, $J=8.8 \mathrm{~Hz}, 1 \mathrm{H}$, benzene-H), 7.10 (t, $J=7.8 \mathrm{~Hz}, 1 \mathrm{H}$, benzene-H), $4.85\left(\mathrm{~d}, J=2.4 \mathrm{~Hz}, 2 \mathrm{H}, \mathrm{CH}_{2}\right), 2.59(\mathrm{t}, J=2.4 \mathrm{~Hz}, 1 \mathrm{H} \mathrm{CH}) .{ }^{13} \mathrm{C}-\mathrm{NMR}$ $\left(100 \mathrm{MHz}, \mathrm{CDCl}_{3}\right) \delta: 150.76,140.35,134.02,125.76,121.45,115.49,77.42,76.78,57.20$.

\subsection{Synthesis of 4-((4-((2-Nitrophenoxy)methyl)-1H-1,2,3-triazol-1-yl)methyl) Benzonitrile (8)}

A solution (260 mL) of 3 prepared from 4-cyanobenzyl bromide (39.20 g, $200 \mathrm{mmol}$ ) was added to a $500-\mathrm{mL}$ round bottom flask, followed by the evaporation of the solvent under reduced pressure. 1-Nitro-2-(prop-2-ynyloxy) benzene (7, $35.4 \mathrm{~g}, 200 \mathrm{mmol})$ was dissolved in $t$ - $\mathrm{BuOH}(300 \mathrm{~mL})$. The solution of 7 and distilled water $(100 \mathrm{~mL})$ was mixed with the residue of $\mathbf{3}$ in the flask and followed by the addition of sodium ascorbate $(250 \mathrm{mg}, 1.26 \mathrm{mmol}$, in $3 \mathrm{~mL}$ of water $)$ and cupric sulfate $\left(\mathrm{CuSO}_{4} \cdot 5 \mathrm{H}_{2} \mathrm{O}\right.$, $85 \mathrm{mg}, 0.34 \mathrm{mmol}$, in $2 \mathrm{~mL}$ of water). After stirring at $60{ }^{\circ} \mathrm{C}$ for $24 \mathrm{~h}$, the mixture was allowed to cool to room temperature. Compound $8(58.21 \mathrm{~g}, 82 \%)$ was obtained as a white solid after the evaporation of about half of the solvent, filtration and drying at $110^{\circ} \mathrm{C}$, m.p. $136-138^{\circ} \mathrm{C} .{ }^{1} \mathrm{H}-\mathrm{NMR}(500 \mathrm{MHz}, \mathrm{DMSO}) \delta$ : $8.36(\mathrm{~s}, 1 \mathrm{H}$, triazole-H), $7.86(\mathrm{~m}, 2 \mathrm{H}$, benzene-H), $7.67(\mathrm{t}, J=7.4 \mathrm{~Hz}, 1 \mathrm{H}$, benzene- $\mathrm{H}), 7.58(\mathrm{~d}, J=8.4 \mathrm{~Hz}$, 1H, benzene-H), 7.46 (d, $J=8.1 \mathrm{~Hz}, 2 \mathrm{H}$, benzene-H), 7.15 (t, $J=7.7 \mathrm{~Hz}, 1 \mathrm{H}$, benzene-H), 5.77 (s, 2H, $\mathrm{CH}_{2}$ ), 5.38 (s, $\left.2 \mathrm{H}, \mathrm{CH}_{2}\right) .{ }^{13} \mathrm{C}-\mathrm{NMR}(125 \mathrm{MHz}, \mathrm{DMSO}) \delta: 151.01,142.73,141.86,140.31,134.76,133.21$, $129.16,125.85,125.40,121.50,118.99,116.21,111.45,63.08,52.70$. 
3.7. General Procedure for the Synthesis of 4-((4-((2-Nitrophenoxy)methyl)-1H-1,2,3 -triazol-1-yl)methyl)-benzamidines $\mathbf{9 a - e}$

A suspension of 8 (3.55 g, $10 \mathrm{mmol})$, in absolute ethanol $(30 \mathrm{~mL})$ was saturated with anhydrous $\mathrm{HCl}$ under water-ice bath cooling; the solution was then stirred at room temperature for 4-5 days. After evaporating most of the solvent, the residue was diluted with dry ether, and the resultant solid obtained by filtration was dissolved in dry methanol. The corresponding amines (40 mmol) were added to the solution and stirred for $24 \mathrm{~h}$ under anhydrous conditions. Then amidines $\mathbf{9 a}-\mathbf{d}$ were treated with the same manner described for compound 4 . Compounds 9 were purified by column chromatography on silica gel, using dichloromethane/methanol $(7: 1, \mathrm{v} / \mathrm{v})$ as the eluent.

4-((4-((2-Nitrophenoxy)methyl)-1H-1,2,3-triazol-1-yl)methyl)-N-butylbenzamidine (9a): Yield, 51.2\%; yellow crystals; m.p. $119-121{ }^{\circ} \mathrm{C} .{ }^{1} \mathrm{H}-\mathrm{NMR}$ (400 MHz, MeOD) $\delta: 8.25$ (s, 1H, triazole-H), 7.77 (d, $J=8.3 \mathrm{~Hz}, 1 \mathrm{H}$, benzene-H), $7.75(\mathrm{~d}, J=8.3 \mathrm{~Hz}, 2 \mathrm{H}$, benzene-H), 7.65-7.60 (m, 1H, benzene-H), $7.60-7.56(\mathrm{~m}, 1 \mathrm{H}$, benzene-H), $7.53(\mathrm{~d}, J=8.2 \mathrm{~Hz}, 2 \mathrm{H}$, benzene-H), $7.11(\mathrm{t}, J=7.7 \mathrm{~Hz}, 1 \mathrm{H}$, benzene-H), $5.77\left(\mathrm{~s}, 2 \mathrm{H}, \mathrm{CH}_{2}\right), 5.34\left(\mathrm{~s}, 2 \mathrm{H}, \mathrm{CH}_{2}\right), 3.46\left(\mathrm{t}, \mathrm{J}=7.2 \mathrm{~Hz}, 2 \mathrm{H}, \mathrm{N}-\mathrm{CH}_{2}\right), 1.74\left(\mathrm{~m}, 2 \mathrm{H}, \mathrm{CH}_{2}\right), 1.48(\mathrm{~m}, 2 \mathrm{H}$, $\left.\mathrm{CH}_{2}\right), 1.01\left(\mathrm{t}, \mathrm{J}=7.3 \mathrm{~Hz}, 3 \mathrm{H}, \mathrm{CH}_{2}\right) .{ }^{13} \mathrm{C}-\mathrm{NMR}(100 \mathrm{MHz}, \mathrm{MeOD}) \delta: 166.02,153.22,143.27,142.65$, $131.41,130.87,130.73,130.68,130.49,127.22,127.06,123.17,117.67,64.74,55.05,41.37,31.38$, 21.51, 14.75. MS (ESI $\left.{ }^{+}\right) \mathrm{m} / z: 409[\mathrm{M}+\mathrm{H}]^{+}$. Anal. Calc. for $\mathrm{C}_{21} \mathrm{H}_{24} \mathrm{~N}_{6} \mathrm{O}_{3} \cdot \mathrm{HCl}$ (444.17): C, 56.69; $\mathrm{H}, 5.66$; N, 18.89; found: C, 56.78; H, 5.81; N, 18.81 .

4-((4-((2-Nitrophenoxy)methyl)-1H-1,2,3-triazol-1-yl)methyl)-N-isobutylbenzamidine (9b): Yield, 36.5\%; white solid; m.p. ${ }^{109-110}{ }^{\circ} \mathrm{C} .{ }^{1} \mathrm{H}-\mathrm{NMR}(400 \mathrm{MHz}, \mathrm{MeOD}) \delta: 8.22(\mathrm{~s}, 1 \mathrm{H}$, triazole-H), 7.79-7.74 (m, 1H, benzene-H), 7.73 (d, $J=8.2 \mathrm{~Hz}, 2 \mathrm{H}$, benzene-H), 7.60 (t, $J=7.3 \mathrm{~Hz}, 1 \mathrm{H}$, benzene-H), 7.52 (d, $J=8.2 \mathrm{~Hz}, 2 \mathrm{H}$, benzene-H), $7.09\left(\mathrm{t}, J=7.6 \mathrm{~Hz}, 1 \mathrm{H}\right.$, benzene-H), $5.75\left(\mathrm{~s}, 2 \mathrm{H}, \mathrm{CH}_{2}\right), 5.32\left(\mathrm{~s}, 2 \mathrm{H}, \mathrm{CH}_{2}\right)$, 3.30-3.22 (d, 2H, N-CH $), 2.06(\mathrm{~m}, 1 \mathrm{H}, \mathrm{CH}), 1.02\left(\mathrm{~d}, J=6.9 \mathrm{~Hz}, 6 \mathrm{H}, \mathrm{CH}_{3}\right) .{ }^{13} \mathrm{C}-\mathrm{NMR}(100 \mathrm{MHz}$, MeOD) $\delta: 166.36,153.23,143.28,143.31,136.12,131.53,130.88,130.72,130.48,127.15,127.06$, 123.17, 117.66, 64.76, 55.06, 52.30, 29.43, 21.25. MS $\left(\mathrm{ESI}^{+}\right) \mathrm{m} / z: 409[\mathrm{M}+\mathrm{H}]^{+}$. Anal. Calc. for $\mathrm{C}_{21} \mathrm{H}_{24} \mathrm{~N}_{6} \mathrm{O}_{3} \cdot \mathrm{HCl}$ (444.17): C, 56.69; H, 5.66; N, 18.89; found: C, 56.73; H, 5.71; N, 18.82 .

4-((4-((2-Nitrophenoxy)methyl)-1H-1,2,3-triazol-1-yl)methyl)-N,N-diethylbenzamidine (9c): Yield, 45.2\%; white solid; m.p. ${ }^{135-137{ }^{\circ} \mathrm{C} . ~}{ }^{1} \mathrm{H}-\mathrm{NMR}(400 \mathrm{MHz}, \mathrm{MeOD}) \delta$ : $8.21(\mathrm{~s}, 1 \mathrm{H}$, triazole-H), 7.76-7.73 (m, 1H, benzene-H), $7.71(\mathrm{~d}, J=8.2 \mathrm{~Hz}, 2 \mathrm{H}$, benzene-H), 7.60 (t, $J=7.3 \mathrm{~Hz}, 1 \mathrm{H}$, benzene-H), $7.52(\mathrm{~d}, J=8.2 \mathrm{~Hz}$, 2H, benzene-H), 7.45 (d, $J=8.4 \mathrm{~Hz}, 1 \mathrm{H}$, benzene-H), 7.10 (t, $J=7.6 \mathrm{~Hz}, 1 \mathrm{H}$, benzene-H), $5.74(\mathrm{~s}, 2 \mathrm{H}$, $\left.\mathrm{CH}_{2}\right), 5.31\left(\mathrm{~s}, 2 \mathrm{H}, \mathrm{CH}_{2}\right), 3.09$ (q, J = $\left.7.3 \mathrm{~Hz}, 4 \mathrm{H}, \mathrm{N}-\mathrm{CH}_{2}\right), 1.31\left(\mathrm{t}, \mathrm{J}=7.3 \mathrm{~Hz}, 6 \mathrm{H}, \mathrm{CH}_{3}\right) .{ }^{13} \mathrm{C}-\mathrm{NMR}$ (100 MHz, MeOD) $\delta: 166.33,153.20,143.99,142.71,136.11,130.71,130.52,130.18,127.04,123.18$, 117.75, 64.76, 55.23, 44.41, 12.47. MS (ESI $\left.{ }^{+}\right) \mathrm{m} / z$ : $409[\mathrm{M}+\mathrm{H}]^{+}$. Anal. Calc. for $\mathrm{C}_{21} \mathrm{H}_{24} \mathrm{~N}_{6} \mathrm{O}_{3} \cdot \mathrm{HCl}(444.17)$ : C, 56.69; H, 5.66; N, 18.89; found: C, 56.81; H, 5.69; N, 18.78 .

4-((4-((2-Nitrophenoxy)methyl)-1H-1,2,3-triazol-1-yl)methyl)-N-cyclohexylbenzamidine (9d): Yield, 57.0\%; white crystals; m.p. $110-113{ }^{\circ} \mathrm{C} .{ }^{1} \mathrm{H}-\mathrm{NMR}(400 \mathrm{MHz}, \mathrm{MeOD}) \delta: 8.17$ (s, 1H, triazole-H), 7.77-7.71 (m, 1H, benzene-H), 7.67 (d, $J=8.2 \mathrm{~Hz}, 2 \mathrm{H}$, benzene-H), 7.60-7.53 (m, 1H, benzene-H), 7.48 (d, $J=8.2 \mathrm{~Hz}, 2 \mathrm{H}$, benzene-H), $7.42(\mathrm{~d}, J=8.4 \mathrm{~Hz}, 1 \mathrm{H}$, benzene-H), 7.07 (t, $J=7.6 \mathrm{~Hz}, 1 \mathrm{H}$, benzene-H), 
$5.72\left(\mathrm{~s}, 2 \mathrm{H}, \mathrm{CH}_{2}\right), 5.30\left(\mathrm{~s}, 2 \mathrm{H}, \mathrm{CH}_{2}\right), 3.70-3.59(\mathrm{~m}, 1 \mathrm{H}, \mathrm{N}-\mathrm{CH}), 1.51-1.35\left(\mathrm{~m}, 5 \mathrm{H}, \mathrm{CH}_{2}\right), 1.16(\mathrm{~m}, 5 \mathrm{H}$, $\left.\mathrm{CH}_{2}\right) .{ }^{13} \mathrm{C}-\mathrm{NMR}(100 \mathrm{MHz}, \mathrm{MeOD}) \delta: 165.09,153.26,145.33,143.21,136.09,131.79,131.45,130.63$, 130.59, 127.07, 123.18, 117.64, 64.79, 55.07, 52.41, 32.76, 26.62, 26.21. MS (ESI $\left.{ }^{+}\right) \mathrm{m} / z: 435[\mathrm{M}+\mathrm{H}]^{+}$. Anal. Calc. for $\mathrm{C}_{19} \mathrm{H}_{29} \mathrm{~N}_{5} \mathrm{O} \cdot \mathrm{HCl}(470.18)$ : C, 58.66; H, 5.78; Cl, 7.53; N, 17.84; found: $\mathrm{C}, 58.78 ; \mathrm{H}$, $5.80 ; \mathrm{N}, 17.91$.

4-((4-((2-Nitrophenoxy)methyl)-1H-1,2,3-triazol-1-yl)methyl)-N,N-dibutylbenzamidine (9e): Yield, 54.6\%; light yellow crystals; m.p. $119-121{ }^{\circ} \mathrm{C} .{ }^{1} \mathrm{H}-\mathrm{NMR}(400 \mathrm{MHz}, \mathrm{MeOD}) \delta: 8.10$ (s, 1H, triazole-H), $7.82(\mathrm{~d}$, $J=8.2 \mathrm{~Hz}, 2 \mathrm{H}$, benzene-H), $7.77(\mathrm{~m}, 1 \mathrm{H}$, benzene-H), $7.57(\mathrm{t}, J=7.4 \mathrm{~Hz}, 1 \mathrm{H}$, benzene-H), $7.41(\mathrm{~d}, J=8.5$ $\mathrm{Hz}, 1 \mathrm{H}$, benzene-H), $7.34(\mathrm{~d}, J=8.2 \mathrm{~Hz}, 2 \mathrm{H}$, benzene-H), $7.08(\mathrm{t}, J=7.7 \mathrm{~Hz}, 1 \mathrm{H}$, benzene-H), $5.67(\mathrm{~s}, 2 \mathrm{H}$, $\left.\mathrm{CH}_{2}\right), 5.32\left(\mathrm{~s}, 2 \mathrm{H}, \mathrm{CH}_{2}\right), 4.23\left(\mathrm{t}, J=6.9 \mathrm{~Hz}, 4 \mathrm{H}, \mathrm{N}-\mathrm{CH}_{2}\right), 3.59\left(\mathrm{~m}, 4 \mathrm{H}, \mathrm{CH}_{2}\right), 1.48-1.29\left(\mathrm{~m}, 4 \mathrm{H}, \mathrm{CH}_{2}\right), 0.97$ $\left(\mathrm{t}, J=7.4 \mathrm{~Hz}, 6 \mathrm{H}, \mathrm{CH}_{3}\right) .{ }^{13} \mathrm{C}-\mathrm{NMR}(100 \mathrm{MHz}, \mathrm{MeOD}) \delta: 165.09,153.01,145.11,143.44,135.75,130.70$, 129.83, 129.58, 126.91, 122.98, 117.44, 64.60, 55.01, 52.25, 29.98, 21.43, 19.98. MS (ESI $\left.{ }^{+}\right) \mathrm{m} / z: 465$ $[\mathrm{M}+\mathrm{H}]^{+}$. Anal. Calc. for $\mathrm{C}_{19} \mathrm{H}_{29} \mathrm{~N}_{5} \mathrm{O} \cdot \mathrm{HCl}(500.23)$ : C, 59.93; H, 6.64; N, 16.77; found: C, 59. 87; H, $6.73 ; \mathrm{N}, 16.72$.

\subsection{Synthesis of 1-Nitro-4-(prop-2-ynyloxy)benzene (12) [51]}

Compound 12 was prepared following the same procedure used for 7. Yield, 76\%; m.p. $118-119{ }^{\circ} \mathrm{C}$; ${ }^{1} \mathrm{H}-\mathrm{NMR}\left(400 \mathrm{MHz}, \mathrm{CDCl}_{3}\right) \delta: 8.22(\mathrm{~d}, J=9.3 \mathrm{~Hz}, 2 \mathrm{H}$, benzene-H), 7.06 (d, $J=9.3 \mathrm{~Hz}, 2 \mathrm{H}$, benzene-H), $4.81\left(\mathrm{~d}, J=2.4 \mathrm{~Hz}, 2 \mathrm{H}, \mathrm{CH}_{2}\right), 2.60(\mathrm{~s}, 1 \mathrm{H}, \mathrm{CH}) .{ }^{13} \mathrm{C}-\mathrm{NMR}\left(100 \mathrm{MHz}, \mathrm{CDCl}_{3}\right) \delta: 162.35,125.85,114.99$, $77.42,77.10,76.80,56.31$.

\subsection{Synthesis of 3-(Azidomethyl)benzonitrile (14)}

3-(Bromomethyl)benzonitrile (13, $39.2 \mathrm{~g}, 200 \mathrm{mmol})$ was dissolved in DMF (180 $\mathrm{mL})$ in an Erlenmeyer flask equipped with a thermometer, then $\mathrm{NaN}_{3}(16.50 \mathrm{~g}, 250 \mathrm{mmol}$, in $90 \mathrm{~mL}$ of distilled water) was added in portions under water-ice bath cooling while stirring, keeping the mixture under $10{ }^{\circ} \mathrm{C}$. After the addition of the azide, the mixture was stirred for $1.5 \mathrm{~h}$ at $30{ }^{\circ} \mathrm{C}$ when the color of the reaction mixture turned colorless and TLC indicated no $\mathbf{1 3}$ remained. The mixture was diluted with toluene $(180 \mathrm{~mL})$ and distilled water $(500 \mathrm{~mL})$ in turn. The aqueous phase was extracted with toluene $(2 \times 60 \mathrm{~mL})$ and the organic phase was combined and dried with $\mathrm{MgSO}_{4}$. After removing of the solvent, 14 (39.6 g, 97\%) was obtained as a white solid; m.p. 30-31 ${ }^{\circ} \mathrm{C} .{ }^{1} \mathrm{H}-\mathrm{NMR}\left(500 \mathrm{MHz}, \mathrm{CDCl}_{3}\right) \delta$ : 7.62-7.57 (m, 2H, benzene-H), 7.57-7.54 (m, 1H, benzene-H), 7.51-7.45 (m, 1H, benzene-H), $4.41(\mathrm{~s}$, $\left.2 \mathrm{H}, \mathrm{CH}_{2}\right) .{ }^{13} \mathrm{C}-\mathrm{NMR}\left(125 \mathrm{MHz}, \mathrm{CDCl}_{3}\right) \delta: 137.25,132.41,131.80,131.38,129.73,118.49$, $112.85,53.62$.

\subsection{General Procedure for the Synthesis of 3-(Azidomethyl)benzamidines 15a-d [42]}

Absolute ethanol $(80 \mathrm{~mL})$ was added to the solution $(25 \mathrm{~mL})$ of $\mathbf{1 4}$. The mixture was sealed and stirred for 7 days at room temprature after being saturated with anhydrous $\mathrm{HCl}$ at ice-water bath. The residue was recrystallized from ether, and then dissolved in absolute methanol $(30 \mathrm{~mL})$. The solution was stirred at $30{ }^{\circ} \mathrm{C}$ overnight after the corresponding amines $(43 \mathrm{mmol})$ were added. After evaporating most of the solvent, the residue was mixed with ethanol $(5 \mathrm{~mL})$ and $2 \mathrm{~N}$ aqueous $\mathrm{NaOH}$ 
$(5 \mathrm{~mL})$. Ether $(50 \mathrm{~mL})$ was added after the aqueous phase being removed, and the target amidines 15a-d was obtained by precipitation.

3-(Azidomethyl)-N-butylbenzamidine (15a): Yield, 75.3\%; yellow crystals; m.p. 106-109 ${ }^{\circ} \mathrm{C} .{ }^{1} \mathrm{H}-\mathrm{NMR}$ (400 MHz, MeOD) $\delta: 7.67$ (s, 1H, benzene-H), $7.64(\mathrm{~d}, J=3.9 \mathrm{~Hz}, 2 \mathrm{H}$, benzene-H), 7.57 (t, $J=7.6 \mathrm{~Hz}$, $1 \mathrm{H}$, benzene-H), $4.48\left(\mathrm{~s}, J=6.3 \mathrm{~Hz}, 2 \mathrm{H}, \mathrm{CH}_{2}\right), 3.41\left(\mathrm{t}, J=7.3 \mathrm{~Hz}, 2 \mathrm{H}, \mathrm{N}-\mathrm{CH}_{2}\right), 1.75-1.52\left(\mathrm{~m}, 2 \mathrm{H}, \mathrm{CH}_{2}\right)$, 1.50-1.30 (m, 2H, $\left.\mathrm{CH}_{2}\right), 0.94\left(\mathrm{t}, 7.4 \mathrm{~Hz}, 3 \mathrm{H}, \mathrm{CH}_{3}\right) .{ }^{13} \mathrm{C}-\mathrm{NMR}(100 \mathrm{MHz}, \mathrm{MeOD}) \delta:$ 164.17, 137.64,

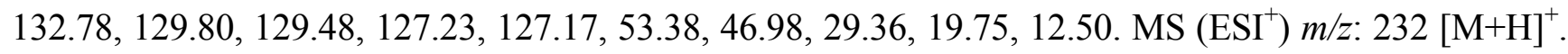
Anal. Calc. for $\mathrm{C}_{12} \mathrm{H}_{17} \mathrm{~N}_{5} \cdot \mathrm{HCl}$ (267.13): C, 53.83; H, 6.78; N, 26.16; found: C, 53. 87; H, 6.71; N, 26.18.

N-Sec-butyl-3-(azidomethyl)benzamidine (15b): Yield, 71.2\%; yellow crystals; m.p. $108-110{ }^{\circ} \mathrm{C}$. ${ }^{1} \mathrm{H}-\mathrm{NMR}$ (400 MHz, DMSO) $\delta$ : 9.64 (s, 2H, N-H), 8.07 (s, 1H, benzene-H), 7.98 (d, J= 7.7 Hz, 2H, benzene-H), 7.89 (t, $J=7.7 \mathrm{~Hz}, 1 \mathrm{H}$, benzene-H), $4.38\left(\mathrm{~s}, 2 \mathrm{H}, \mathrm{CH}_{2}\right), 3.28(\mathrm{~m}, 1 \mathrm{H}, \mathrm{N}-\mathrm{CH}), 1.85(\mathrm{~m}, 2 \mathrm{H}$, $\left.\mathrm{CH}_{2}\right), 1.45\left(\mathrm{~d}, J=6.6 \mathrm{~Hz}, 3 \mathrm{H}, \mathrm{CH}_{3}\right), 1.21\left(\mathrm{t}, J=7.4 \mathrm{~Hz}, 3 \mathrm{H}, \mathrm{CH}_{3}\right) .{ }^{13} \mathrm{C}-\mathrm{NMR}(100 \mathrm{MHz}, \mathrm{DMSO}) \delta$ : $162.39,136.98,133.17,130.16,129.62,128.65,128.53,53.38,51.12,28.59,19.65,10.83 . \mathrm{MS}_{(\mathrm{ESI}}^{+}$) m/z: $232[\mathrm{M}+\mathrm{H}]^{+}$. Anal. Calc. for $\mathrm{C}_{12} \mathrm{H}_{17} \mathrm{~N}_{5} \cdot \mathrm{HCl}$ (267.13): C, 53.83; H, 6.78; N, 26.16; found: C, 53. $85 ; \mathrm{H}, 6.82 ; \mathrm{N}, 26.19$.

3-(Azidomethyl)-N-cyclohexylbenzamidine (15c): Yield, 78.0\%; white crystals; m.p. 110-111 ${ }^{\circ} \mathrm{C}$. ${ }^{1} \mathrm{H}-\mathrm{NMR}$ (400 MHz, MeOD) $\delta: 7.77$ (s, 1H, benzene-H), $7.59(\mathrm{~d}, J=7.7 \mathrm{~Hz}, 2 \mathrm{H}$, benzene-H), 7.39 (t, $J=7.7 \mathrm{~Hz}, 1 \mathrm{H}$, benzene-H), $4.42\left(\mathrm{~s}, 2 \mathrm{H}, \mathrm{CH}_{2}\right), 2.94-2.79(\mathrm{~m}, 1 \mathrm{H}, \mathrm{N}-\mathrm{CH}), 1.91\left(\mathrm{~m}, 5 \mathrm{H}, \mathrm{CH}_{3}\right)$, $1.80-1.65\left(\mathrm{~m}, 5 \mathrm{H}, \mathrm{CH}_{3}\right) .{ }^{13} \mathrm{C}-\mathrm{NMR}(100 \mathrm{MHz}, \mathrm{MeOD}) \delta: 163.08,137.52,132.67,131.37,130.09$, 129.41, 127.31, 127.22, 53.39, 48.28, 31.29, 24.80. MS $\left(\mathrm{ESI}^{+}\right) \mathrm{m} / z: 258[\mathrm{M}+\mathrm{H}]^{+}$. Anal. Calc. for $\mathrm{C}_{14} \mathrm{H}_{19} \mathrm{~N}_{5} \cdot \mathrm{HCl}$ (293.14): C, 57.23; H, 6.86; Cl, 12.07; N, 23.84; found: C, 57. 35; H, 6.81; N, 26.20.

3-(Azidomethyl)-N,N-diethylbenzamidine (15d): Yield, 55.4\%; white crystals; m.p. $99-101{ }^{\circ} \mathrm{C} .{ }^{1} \mathrm{H}-\mathrm{NMR}$ (400 MHz, DMSO) $\delta: 9.44(\mathrm{~s}, 3 \mathrm{H}, \mathrm{N}-\mathrm{H}), 8.10$ (s, 1H, benzene-H), 7.93 (d, $J=7.7 \mathrm{~Hz}, 2 \mathrm{H}$, benzene-H), $7.87\left(\mathrm{t}, J=7.7 \mathrm{~Hz}, 1 \mathrm{H}\right.$, benzene-H), $4.45\left(\mathrm{~s}, 2 \mathrm{H}, \mathrm{CH}_{2}\right), 2.82-2.75\left(\mathrm{~m}, 4 \mathrm{H}, \mathrm{N}-\mathrm{CH}_{2}\right), 1.16$ (t, $\left.J=7.4 \mathrm{~Hz}, 6 \mathrm{H}, \mathrm{CH}_{3}\right) . \mathrm{MS}\left(\mathrm{ESI}^{+}\right) \mathrm{m} / \mathrm{z}: 232[\mathrm{M}+\mathrm{H}]^{+}$. Anal. Calc. for $\mathrm{C}_{12} \mathrm{H}_{17} \mathrm{~N}_{5} \cdot \mathrm{HCl}$ (267.13): C, 53.83; H, 6.78; N, 26.16; found: C, 53. 86; H, 6.75; N, 26.14 .

\subsection{General Procedure for the Synthesis of 3-((4-(Hydroxymethyl)-1H-1,2,3-triazol-1-yl)methyl)- benzamidines $\mathbf{1 6 a}-\mathbf{d}$}

Compound 15 (4.5 mmol) and propargyl alcohol $(28 \mathrm{mg}, 5 \mathrm{mmol})$ was dissolved in a mixture of $t$-butanol $(30 \mathrm{~mL})$ and distilled water $(12 \mathrm{~mL})$, and then, ascorbate sodium $(45 \mathrm{mg}, 0.23 \mathrm{mmol}$, in $1 \mathrm{~mL}$ water) and $\mathrm{CuSO}_{4} \cdot 5 \mathrm{H}_{2} \mathrm{O}(10 \mathrm{mg}, 0.04 \mathrm{mmol}$, in $1 \mathrm{~mL}$ water $)$ were added to the mixture. After stirring at $60{ }^{\circ} \mathrm{C}$ for $48 \mathrm{~h}$, half of the solvent was evaporated and the target molecule was obtained by recrystallization from ether (16a) or column chromatography on silica gel (16b, 16c, 16d), using dichloromethane/methanol $(5: 1, \mathrm{v} / \mathrm{v})$ as the eluent.

N-Butyl-3-((4-(hydroxymethyl)-1H-1,2,3-triazol-1-yl)methyl)benzamidine (16a): Yield, 68.8\%; light yellow crystals; m.p. $106-109^{\circ} \mathrm{C} .{ }^{1} \mathrm{H}-\mathrm{NMR}(400 \mathrm{MHz}, \mathrm{MeOD}) \delta: 8.07$ (s, 1H, triazole-H), 7.80 (s, 1H, benzene-H), $7.66(\mathrm{~d}, J=7.7 \mathrm{~Hz}, 2 \mathrm{H}$, benzene-H), $7.59(\mathrm{t}, J=7.7 \mathrm{~Hz}, 1 \mathrm{H}$, benzene-H), $5.72(\mathrm{~s}, 2 \mathrm{H}$, 
$\left.\mathrm{CH}_{2}\right), 4.65$ (s, 2H, CH ), $3.46\left(\mathrm{t}, 2 \mathrm{H}, \mathrm{N}-\mathrm{CH}_{2}\right), 1.79-1.68\left(\mathrm{~m}, 2 \mathrm{H}, \mathrm{CH}_{2}\right), 1.48\left(\mathrm{~m}, 2 \mathrm{H}, \mathrm{CH}_{2}\right), 0.99$ (m, $\left.3 \mathrm{H}, \mathrm{CH}_{3}\right) .{ }^{13} \mathrm{C}-\mathrm{NMR}(100 \mathrm{MHz}, \mathrm{DMSO}) \delta$ : 163.18, 136.16, 132.01, 129.11, 128.93, 126.94, 126.64, 122.58, 54.22, 51.94, 42.09, 38.35, 18.50, 11.92. $\mathrm{MS}\left(\mathrm{ESI}^{+}\right) \mathrm{m} / z: 288[\mathrm{M}+\mathrm{H}]^{+}$. Anal. Calc. for $\mathrm{C}_{15} \mathrm{H}_{21} \mathrm{~N}_{5} \mathrm{O} \cdot \mathrm{HCl}(323.15): \mathrm{C}, 55.64 ; \mathrm{H}, 6.85 ; \mathrm{N}, 21.63$; found: $\mathrm{C}, 55.41 ; \mathrm{H}, 6.72 ; \mathrm{N}, 21.57$.

N-Sec-butyl-3-((4-(hydroxymethyl)-1H-1,2,3-triazol-1-yl)methyl)benzamidine (16b): Yield, 71.1\%; light yellow crystals; m.p. $108-110{ }^{\circ} \mathrm{C} .{ }^{1} \mathrm{H}-\mathrm{NMR}(400 \mathrm{MHz}, \mathrm{MeOD}) \delta: 8.05$ (s, 1H, triazole-H), 7.72 (s, 1H, benzene-H), 7.67 (d, $J=7.7 \mathrm{~Hz}, 2 \mathrm{H}$, benzene-H), 7.59 (t, $J=7.6 \mathrm{~Hz}, 1 \mathrm{H}$, benzene-H), $5.71\left(\mathrm{~s}, 2 \mathrm{H}, \mathrm{CH}_{2}\right)$, $4.64\left(\mathrm{~s}, 2 \mathrm{H}, \mathrm{CH}_{2}\right), 3.81$ (t, J = 7.4 Hz, 1H, N-H), 1.75-1.64 (m, 2H, $\left.\mathrm{CH}_{2}\right), 1.32\left(\mathrm{~m}, 2 \mathrm{H}, \mathrm{CH}_{2}\right), 1.01$ (t, $\left.J=7.4 \mathrm{~Hz}, 3 \mathrm{H}, \mathrm{CH}_{3}\right) .13 \mathrm{C}-\mathrm{NMR}(100 \mathrm{MHz}, \mathrm{DMSO}) \delta$ : 162.68, 136.11, 131.94, 129.40, 128.89, 126.94, 126.63, 122.54, 54.22, 51.92, 50.56, 26.58, 17.26, 16.06. MS (ESI $\left.{ }^{+}\right) m / z: 288[\mathrm{M}+\mathrm{H}]^{+}$. Anal. Calc. for $\mathrm{C}_{15} \mathrm{H}_{21} \mathrm{~N}_{5} \mathrm{O} \cdot \mathrm{HCl}(323.15): \mathrm{C}, 55.64 ; \mathrm{H}, 6.85 ; \mathrm{N}, 21.63$; found: C, 55.42; H, 6.78; N, 21.71.

N-Cyclohexyl-3-((4-(hydroxymethyl)-1H-1,2,3-triazol-1-yl)methyl)benzamidine (16c): Yield, 76.2\%; light yellow crystals; m.p. $110-11{ }^{\circ} \mathrm{C} .{ }^{1} \mathrm{H}-\mathrm{NMR}$ (400 MHz, DMSO) $\delta$ : 7.95 (s, 1H, triazole-H), 7.63 (s, 1H, benzene-H), 7.57 (d, $J=7.8 \mathrm{~Hz}, 2 \mathrm{H}$, benzene-H), 7.49 (t, $J=7.6 \mathrm{~Hz}, 1 \mathrm{H}$, benzene-H), 5.61 (s, $\left.2 \mathrm{H}, \mathrm{CH}_{2}\right), 4.55\left(\mathrm{~s}, 2 \mathrm{H}, \mathrm{CH}_{2}\right), 3.59(\mathrm{~m}, 1 \mathrm{H}, \mathrm{N}-\mathrm{H}), 1.44-1.29\left(\mathrm{~m}, 5 \mathrm{H}, \mathrm{CH}_{2}\right), 1.24\left(\mathrm{~m}, 5 \mathrm{H}, \mathrm{CH}_{2}\right)$. ${ }^{13} \mathrm{C}-\mathrm{NMR}(100 \mathrm{MHz}, \mathrm{DMSO}) \delta$ : 162.12, 136.04, 131.92, 129.39, 128.84, 126.73, 126.55, 122.46, 54.22, 52.34, 51.92, 51.88, 46.20, 30.30, 23.98. $\mathrm{MS}\left(\mathrm{ESI}^{+}\right) \mathrm{m} / z: 314[\mathrm{M}+\mathrm{H}]^{+}$. Anal. Calc. for $\mathrm{C}_{17} \mathrm{H}_{23} \mathrm{~N}_{5} \mathrm{O} \cdot \mathrm{HCl}$ (349.17): C, 58.36; H, 6.91; N, 20.02; found: C, 58.47; H, 6.89; N, 20.13.

N,N-Diethyl-3-((4-(hydroxymethyl)-1H-1,2,3-triazol-1-yl)methyl)benzamidine (16d): Yield, 70.3\%; light yellow crystals; m.p. 99-101 ${ }^{\circ} \mathrm{C} .{ }^{1} \mathrm{H}-\mathrm{NMR}(400 \mathrm{MHz}, \mathrm{MeOD}) \delta: 8.09$ (s, 1H, triazole-H), $7.65(\mathrm{~d}$, $J=7.4 \mathrm{~Hz}, 1 \mathrm{H}$, benzene-H), 7.62 (d, $J=8.1 \mathrm{~Hz}, 1 \mathrm{H}$, benzene-H), 7.55 (m, 2H, benzene-H), 5.74 (s, $\left.2 \mathrm{H}, \mathrm{CH}_{2}\right), 4.66\left(\mathrm{~s}, 2 \mathrm{H}, \mathrm{CH}_{2}\right), 3.33-3.27\left(\mathrm{~m}, 4 \mathrm{H}, \mathrm{N}-\mathrm{CH}_{2}\right), 1.37\left(\mathrm{t}, J=7.2 \mathrm{~Hz}, 6 \mathrm{H}, \mathrm{CH}_{3}\right) .{ }^{13} \mathrm{C}-\mathrm{NMR}(100$ MHz, DMSO) $\delta$ : 163.75, 162.39, 136.43, 130.50, 129.51, 129.04, 126.35, 125.91, 54.24, 51.92, 42.31, 11.63. $\mathrm{MS}\left(\mathrm{ESI}^{+}\right) m / z: 288[\mathrm{M}+\mathrm{H}]^{+}$. Anal. Calc. for $\mathrm{C}_{15} \mathrm{H}_{21} \mathrm{~N}_{5} \mathrm{O} \cdot \mathrm{HCl}(323.15): \mathrm{C}, 55.64 ; \mathrm{H}, 6.85 ; \mathrm{N}$, 21.63; found: C, 55.42; H, 6.78; N, 21.71.

3.12. General Procedure for the Synthesis of 3-((4-((4-Nitrophenoxy)methyl)-1H-1,2,3-triazol-1-yl) methyl)benzamidines $\mathbf{1 7 a - d}$

Compounds $17 \mathbf{a}-\mathbf{d}$ were prepared using the same procedure as for compounds $\mathbf{1 6 a}-\mathbf{d}$, by replacing the propargyl alcohol with 1-nitro-4-(prop-2-ynyloxy)benzene (12).

3-((4-((4-Nitrophenoxy)methyl)-1H-1,2,3-triazol-1-yl)methyl)-N-butylbenzamidine (17a): Yield, 80.1\%; light yellow crystals; m.p. $164-166{ }^{\circ} \mathrm{C} .{ }^{1} \mathrm{H}-\mathrm{NMR}(400 \mathrm{MHz}, \mathrm{MeOD}) \delta: 8.29$ (s, 1H, triazole-H), 8.13 (s, $J=9.1 \mathrm{~Hz}, 2 \mathrm{H}$, benzene-H), $7.74(\mathrm{~s}, 1 \mathrm{H}$, benzene-H), $7.67(\mathrm{~d}, J=7.1 \mathrm{~Hz}, 1 \mathrm{H}$, benzene-H), $7.65(\mathrm{~d}$, $J=7.2 \mathrm{~Hz}, 1 \mathrm{H}$, benzene-H), $7.61(\mathrm{~s}, 1 \mathrm{H}$, benzene-H), $7.11(\mathrm{~d}, J=9.1 \mathrm{~Hz}, 2 \mathrm{H}$, benzene-H), 5.71 (s, 2H, $\left.\mathrm{CH}_{2}\right), 5.24\left(\mathrm{~s}, 2 \mathrm{H}, \mathrm{CH}_{2}\right), 2.93-2.81\left(\mathrm{~m}, 2 \mathrm{H}, \mathrm{N}-\mathrm{CH}_{2}\right), 1.74-1.63\left(\mathrm{~m}, 2 \mathrm{H}, \mathrm{CH}_{2}\right), 1.34\left(\mathrm{~m}, 2 \mathrm{H}, \mathrm{CH}_{2}\right), 0.93$ $\left(\mathrm{d}, J=2.3 \mathrm{~Hz}, 3 \mathrm{H}, \mathrm{CH}_{3}\right) .{ }^{13} \mathrm{C}-\mathrm{NMR}(100 \mathrm{MHz}, \mathrm{DMSO}) \delta: 163.11,162.58,140.85,135.89,132.13$, $132.02,129.12,129.00,128.73,127.04,126.77,126.48,126.40,124.18,114.04,60.80,52.10,42.11$, 
28.56, 18.95, 11.92. $\mathrm{MS}\left(\mathrm{ESI}^{+}\right) \mathrm{m} / z: 409[\mathrm{M}+\mathrm{H}]^{+}$. Anal. Calc. for $\mathrm{C}_{21} \mathrm{H}_{24} \mathrm{~N}_{6} \mathrm{O}_{3} \cdot \mathrm{HCl}$ (444.17): C, 56.69; H, 5.66; N, 18.89; found: C, 56.71; H, 5.72; N, 18.84 .

3-((4-((4-Nitrophenoxy)methyl)-1H-1,2,3-triazol-1-yl)methyl)-N-sec-butylbenzamidine (17b): Yield, 69.6\%; yellow solid; m.p. 92-94 ${ }^{\circ} \mathrm{C} .{ }^{1} \mathrm{H}-\mathrm{NMR}$ (400 MHz, MeOD) $\delta: 8.31$ (s, 1H, triazole-H), 8.17 (d, $J=9.1 \mathrm{~Hz}, 2 \mathrm{H}$, benzene-H), $7.76(\mathrm{~s}, 1 \mathrm{H}$, benzene-H), $7.68(\mathrm{~d}, J=7.1 \mathrm{~Hz}, 1 \mathrm{H}$, benzene-H), $7.66(\mathrm{~d}$, $J=7.2 \mathrm{~Hz}, 1 \mathrm{H}$, benzene-H), $7.60(\mathrm{~m}, 1 \mathrm{H}$, benzene-H), $7.15(\mathrm{~d}, J=9.2 \mathrm{~Hz}, 2 \mathrm{H}$, benzene-H), 5.75 (s, $\left.2 \mathrm{H}, \mathrm{CH}_{2}\right), 5.28\left(\mathrm{~s}, 2 \mathrm{H}, \mathrm{CH}_{2}\right), 3.89-3.75(\mathrm{~m}, 1 \mathrm{H}, \mathrm{N}-\mathrm{CH}), 1.67\left(\mathrm{~m}, 2 \mathrm{H}, \mathrm{CH}_{2}\right), 1.27(\mathrm{~d}, J=6.6 \mathrm{~Hz}, 3 \mathrm{H}$, $\left.\mathrm{CH}_{3}\right), 1.02-0.98\left(\mathrm{~m}, 3 \mathrm{H}, \mathrm{CH}_{3}\right) .{ }^{13} \mathrm{C}-\mathrm{NMR}(100 \mathrm{MHz}, \mathrm{DMSO}) \delta: 162.64,162.57,140.89,135.89$, 132.04, 131.92, 129.43, 128.94, 127.03, 126.77, 126.48, 126.40, 124.08, 113.99, 60.79, 52.05, 50.57, 28.98, 17.25, 8.61. MS (ESI $\left.{ }^{+}\right) \mathrm{m} / z: 409[\mathrm{M}+\mathrm{H}]^{+}$. Anal. Calc. for $\mathrm{C}_{21} \mathrm{H}_{24} \mathrm{~N}_{6} \mathrm{O}_{3} \cdot \mathrm{HCl}$ (444.17): C, 56.69; H, 5.66; N, 18.89; found: C, 56.63; H, 5.61; N, 18.93 .

3-((4-((4-Nitrophenoxy)methyl)-1H-1,2,3-triazol-1-yl)methyl)-N-cyclohexylbenzamidine (17c): Yield, 71.5\%; brown solid; m.p. 101-103 ${ }^{\circ} \mathrm{C} .{ }^{1} \mathrm{H}-\mathrm{NMR}$ (400 MHz, MeOD) $\delta: 8.19$ (s, 1H, triazole-H), 8.05 (d, $J=7.8 \mathrm{~Hz}, 2 \mathrm{H}$, benzene-H), $7.65(\mathrm{~s}, 1 \mathrm{H}$, benzene-H), $7.58(\mathrm{~d}, J=7.1 \mathrm{~Hz}, 1 \mathrm{H}$, benzene-H), $7.56(\mathrm{~d}$, $J=7.1 \mathrm{~Hz}, 1 \mathrm{H}$, benzene-H), $7.48(\mathrm{~d}, J=7.8 \mathrm{~Hz}, 1 \mathrm{H}$, benzene-H), $7.04(\mathrm{~d}, J=7.8 \mathrm{~Hz}, 2 \mathrm{H}$, benzene-H), $5.63\left(\mathrm{~s}, 2 \mathrm{H}, \mathrm{CH}_{2}\right), 5.17\left(\mathrm{~s}, 2 \mathrm{H}, \mathrm{CH}_{2}\right), 3.18(\mathrm{~m}, 1 \mathrm{H}, \mathrm{N}-\mathrm{CH}), 1.55\left(\mathrm{~m}, 5 \mathrm{H}, \mathrm{CH}_{2}\right), 1.32(\mathrm{~d}, J=10.0 \mathrm{~Hz}$, $\left.5 \mathrm{H}, \mathrm{CH}_{2}\right)$. MS $\left(\mathrm{ESI}^{+}\right) \mathrm{m} / z: 435[\mathrm{M}+\mathrm{H}]^{+}$. Anal. Calc. for $\mathrm{C}_{19} \mathrm{H}_{29} \mathrm{~N}_{5} \mathrm{O} \cdot \mathrm{HCl}(470.18): \mathrm{C}, 58.66 ; \mathrm{H}, 5.78$; $\mathrm{Cl}, 7.53 ; \mathrm{N}, 17.84$; found: C, 58.78; H, 5.82; N, 17.81 .

3-((4-((4-Nitrophenoxy)methyl)-1H-1,2,3-triazol-1-yl)methyl)-N,N-diethylbenzamidine (17d): Yield, 68.9\%; brown solid; m.p. $159-161{ }^{\circ} \mathrm{C} .{ }^{1} \mathrm{H}-\mathrm{NMR}$ (400 MHz, MeOD) $\delta: 8.36$ (s, 1H, triazole-H), 8.21 (d, $J=8.9 \mathrm{~Hz}, 2 \mathrm{H}$, benzene-H), 7.68 (s, 1H, benzene-H), 7.64 (m, 2H, benzene-H), 7.54 (d, $J=7.7 \mathrm{~Hz}$, $1 \mathrm{H}$, benzene-H), $7.20\left(\mathrm{~d}, J=8.9 \mathrm{~Hz}, 2 \mathrm{H}\right.$, benzene-H), $5.79\left(\mathrm{~s}, 2 \mathrm{H}, \mathrm{CH}_{2}\right), 5.32\left(\mathrm{~s}, 2 \mathrm{H}, \mathrm{CH}_{2}\right), 3.40-3.25$ $\left(\mathrm{m}, 4 \mathrm{H}, \mathrm{N}-\mathrm{CH}_{2}\right), 1.38\left(\mathrm{t}, J=7.2 \mathrm{~Hz}, 6 \mathrm{H}, \mathrm{CH}_{3}\right) . \mathrm{MS}\left(\mathrm{ESI}^{+}\right) \mathrm{m} / z: 409[\mathrm{M}+\mathrm{H}]^{+}$. Anal. Calc. for $\mathrm{C}_{21} \mathrm{H}_{24} \mathrm{~N}_{6} \mathrm{O}_{3} \cdot \mathrm{HCl}$ (444.17): C, 56.69; H, 5.66; N, 18.89; found: C, 56.72; H, 5.61; N, 19.02 .

\subsection{Fungicidal Activity Bioassay: Effect of the New Compounds on the Mycelial Growth of C. lagenarium and B. cinerea in Solid Media}

The compounds 4a-e, 9a-e, 15a-d, 16a-d, 17a-d were dissolved in ethanol $(5,000 \mu \mathrm{g} / \mathrm{mL})$ and mixed with sterile molten potato dextrose agar (PDA) to obtain final concentrations of $200 \mu \mathrm{g} / \mathrm{mL}$. The commercial fungicide carbendazim SC (with a $50 \%$ composition) was used as the positive control. Fungicidal activities of the compounds against $B$. cinerea were evaluated in vitro using the method given in [52]. The inhibition rate was calculated according to Equation (1):

$$
\mathrm{I}_{1}=\left(\mathrm{D}_{1}-\mathrm{D}_{0}\right) / \mathrm{D}_{1} \times 100 \%
$$

where $I_{1}$ is the inhibition rate, $D_{1}$ is the average diameter of mycelia in the blank test, and $D_{0}$ is the average diameter of mycelia in the presence of compounds. The results are given in Table 1. 


\subsection{Effect on C. lagenarium and B. cinerea Activity on Cucumber Leaves [53]}

Fungicidal activity against Colletotrichum lagenarium and Botrytis cinerea was tested on cucumber (Cucumis sarivus L.) seedlings at three leaf stages. The commercial fungicide carbendazim SC (with a $50 \%$ composition) was adopted as the positive control. Methanol solutions of the compounds and carbendazim $(5,000 \mu \mathrm{g} / \mathrm{mL})$ were diluted to concentrations at $300 \mu \mathrm{g} / \mathrm{mL}$ with $0.1 \%$ PEG400 solution of water to obtain solutions that were spread on the surface of the cucumber leaves. After air drying for $24 \mathrm{~h}$, the upper sides of the leaves were inoculated with $4 \mathrm{~mm}$ plugs of $C$. lagenarium or B. cinerea maintained on PDA. Nine replicates were performed. The inhibition rate was calculated according to Equation (1), and the results are shown in Table 1.

\section{Conclusions}

Eighteen novel benzamidines with 1,2,3-triazole moieties connected to the benzene ring and four benzamidines containing azide groups were synthesized and characterized. Bioassays showed that the synthesized benzamidine compounds had weak antifungal activities against the tested fungi in vitro, however, the activities in vivo of some compounds to the same strains were excellent. Compounds $\mathbf{4 c}$, 9a, 9b, 16d and 15b exhibited significant activities toward $C$. lagenarium in vivo. Compounds $\mathbf{4 b}, \mathbf{4 e}$, and $\mathbf{1 7} \mathbf{a}$ and $\mathbf{1 5 b}$ showed excellent inhibitory activities toward B. cinerea.

\section{Supplementary Materials}

Supplementary materials can be accessed at: http://www.mdpi.com/1420-3049/19/5/5674/s1.

\section{Acknowledgments}

We appreciate the kind help of Hong-li Zhang during the NMR spectroscopic analysis of the compounds synthesized.

\section{Author Contributions}

The work presented was carried out in collaboration between all authors. Guangyou Chen and Xing Zhang defined the research theme. Guangyou Chen and Chonglin Cai designed methods and experiments, carried out the laboratory experiments, analyzed the data, interpreted the results and wrote the paper. Jia Lu and Yiwan Zhou co-designed the synthesis experiments, and co-worked on associated data collection and their interpretation. All authors have contributed to, seen and approved the manuscript.

\section{Conflicts of Interest}

The authors declare no conflict of interest.

\section{References}

1. Russell, P.E. Fungicide resistance-occurrence and management. J. Agr. Sci. 1995, 124, 317-323. 
2. Jarak, I.; Marjanovic, M.; Piantanida, I.; Kralj, M.; Karminski-Zamola, G. Novel pentamidine derivatives: Synthesis, anti-tumor properties and polynucleotide-binding activities. Eur. J. Med. Chem. 2011, 46, 2807-2815.

3. Donkor, I.O.; Assefa, H.; Rattendi, D.; Lane, S.; Vargas, M.; Goldberg, B.; Bacchi, C. Trypanocidal activity of dicationic compounds related to pentamidine. Eur. J. Med. Chem. 2001, $36,531-538$.

4. Werbovetz, K. Diamidines as antitrypanosomal, antileishmanial and antimalarial agents. Curr. Opin. Invest. Dr. 2006, 7, 147-157.

5. Paine, M.F.; Wang, M.Z.; Generaux, C.N.; Boykin, D.W.; Wilson, W.D.; de Koning, H.P.; Olson, C.A.; Pohlig, G.; Burri, C.; Brun, R.; et al. Diamidines for human African trypanosomiasis. Curr. Opin. Invest. Dr. 2010, 11, 876-883.

6. Berger, O.; Kaniti, A.; van Ba, C.T.; Vial, H.; Ward, S.A.; Biagini, G.A.; Bray, P.G.; O'Neill, P.M. Synthesis and antimalarial activities of a diverse set of triazole-containing furamidine analogues. Chem. Med. Chem. 2011, 6, 2094-2108.

7. Kimura, A.; Nishikawa, H.; Nomura, N.; Mitsuyama, J.; Fukumoto, S.; Inoue, N.; Kawazu, S. In vitro and in vivo antimalarial activities of T-2307, a novel arylamidine. Antimicrob. Agents Chemother. 2012, 56, 2191-2193.

8. Donkor, I.O.; Queener, S.F.; Dalton, J.T. Pentamidine congeners. 4. DNA binding affinity and anti-Pneumocystis carinii activity of butamidine analogues. Bioorg. Med. Chem. Lett. 1996, 6, 1967-1970.

9. Arya, S.; Kumar, N.; Roy, P.; Sondhi, S.M. Synthesis of amidine and bis amidine derivatives and their evaluation for anti-inflammatory and anticancer activity. Eur. J. Med. Chem. 2013, 59, 7-14.

10. Del Poeta, M.; Schell, W.A.; Dykstra, C.C.; Jones, S.; Tidwell, R.R.; Czarny, A.; Bajic, M.; Bajic, M.; Kumar, A.; Boykin, D.; et al. Structure in vitro activity relationships of pentamidine analogues and dication-substituted bis-benzimidazoles as new antifungal agents. Antimicrob. Agents Chemother. 1998, 42, 2495-2502.

11. Donkor, I.O.; Clark, A.M. In vitro antimicrobial activity of aromatic diamidines and diimidazolines related to pentamidine. Eur. J. Med. Chem. 1999, 34, 639-643.

12. Afeltra, J.; Meis, J.; Vitale, R.G.; Mouton, J.W.; Verweij, P.E.; Eurofung, N. In vitro activities of pentamidine, pyrimethamine, trimethoprim, and sulfonamides against Aspergillus species. Antimicrob. Agents Chemother. 2002, 46, 2029-2031.

13. Laurent, J.; Stanicki, D.; Huang, T.L.; Dei-Cas, E.; Pottier, M.; Aliouat, E.M.; Eynde, J.J.V. Bisbenzamidines as antifungal agents. Are both amidine functions required to observe an anti-pneumocystis carinii activity? Molecules 2010, 15, 4283-4293.

14. Huang, S.H.; Nesterov, V.N.; Richmond, M.G. New azido-substituted tantalum compounds: Syntheses and DFT examination of nitrogen-rich mono-, di-, and trinuclear tantalum(V) compounds. Dalton Trans. 2014, 43, 3453-3461.

15. Soeiro, M.N.C.; de Souza, E.M.; Stephens, C.E.; Boykin, D.W. Aromatic diamidines as antiparasitic agents. Expert Opin. Inv. Drug. 2005, 14, 957-972.

16. Chen, A.L.; He, J.; Lian, Y.J.; Feng, J.T.; Zhang, X. The fungicide activity of analogs of pentamidine against Botrytis cinerea. Acta Phytophylacica Sin. 2005, 33, 68-72. 
17. Chen, A.L.; Lian, Y.; Ye, H.Y.; Feng, J.T.; Zhang, X. The bioactivity of novel fungicide propamidine against Botrytis cinerea. Chin. Agric. Sci. Bull. 2004, 21, 301-303.

18. Forge, D.; Cappoen, D.; Laurent, J.; Stanicki, D.; Mayence, A.; Huang, T.L.; Verschaeve, L.; Huygen, K.; Vanden Eynde, J.J. 1,4-Diarylpiperazines and analogs as anti-tubercular agents: Synthesis and biological evaluation. Eur. J. Med. Chem. 2012, 49, 95-101.

19. Alp, M.; Goker, H.; Brun, R.; Yildiz, S. Synthesis and antiparasitic and antifungal evaluation of 2'-arylsubstituted-1H,1'H-2,5' bisbenzimidazolyl-5-carboxamidines. Eur. J. Med. Chem. 2009, 44, 2002-2008.

20. Weidner-Wells, M.A.; Ohemeng, K.A.; Nguyen, V.N.; Fraga-Spano, S.; Macielag, M.J.; Werblood, H.M.; Foleno, B.D.; Webb, G.C.; Barrett, J.F.; Hlasta, D.J. Amidino benzimidazole inhibitors of bacterial two-component systems. Bioorg. Med. Chem. Lett. 2001, 11, 1545-1548.

21. Vicentini, C.B.; Brandolini, V.; Guarneri, M.; Giori, P. Pyrazolo[3,4-d][1,2,3]triazole-1carboxamides and 5-alkylaminopyrazolo[3,4-d]oxazoles: Synthesis and evaluation of the in vitro antifungal activity. Farmaco 1992, 47, 1021-1034.

22. Gaur, M.; Goel, M.; Sridhar, L.; Ashok, T.D.S.; Prabhakar, S.; Dureja, P.; Raghunathan, P.; Eswaran, S.V. Synthesis, characterization, and antifungal activity of biaryl-based bis(1,2,3triazoles) using click chemistry. Mon. Chem. 2012, 143, 283-288.

23. Su, N.N.; Li, Y.; Yu, S.J.; Zhang, X.; Liu, X.H.; Zhao, W.G. Microwave-assisted synthesis of some novel 1,2,3-triazoles by click chemistry, and their biological activity. Res. Chem. Intermediat. 2013, 39, 759-766.

24. Alam, M.S.; Ozoe, Y.; Lee, D.U. Structure-antimicrobial activity relationship of 4-or 5-substituted 1-(2,6-Dichloro-4-trifluoromethylphenyl)-1H-1,2,3-triazole analogues. J. Korean Soc. Appl. Biol. Chem. 2011, 54, 149-153.

25. Pereira, D.; Fernandes, P. Synthesis and antibacterial activity of novel 4-aryl-1,2,3-triazole containing macrolides. Bioorg. Med. Chem. Lett. 2011, 21, 510-513.

26. Zhao, Q.; Lu, J.R.; Xin, C.W.; Bao, X.R.; Gao, H.Y.; Zhao, X.; Li, S.; Ru, T.T. Synthesis and antibacterial activities of 1-(4-Substituted phenyl)-4-phenyl-5-(2-hydroxy benzyl) amino-1,2,3triazole derivatives. Chem. J. Chin. Univ. Chin. 2011, 32, 2806-2811.

27. Peng, C.Y.; Xin, C.W.; Li, J.F.; Ji, D.; Bao, X.R.; Lu, J.R. Synthesis and biological activities of 1-(4-Methyl)phenyl-5-substituted phenylimino-1,2,3-triazole carboxylic acid/caboxylic acid amide. Chin. J. Org. Chem. 2013, 33, 383-388.

28. Da Silva, F.D.; de Souza, M.; Frugulhetti, I.I.P.; Castro, H.C.; Souza, S.L.D.; de Souza, T.M.L.; Rodrigues, D.Q.; Souza, A.M.T.; Abreu, P.A.; Passamani, F.; et al. Synthesis, HIV-RT inhibitory activity and SAR of 1-benzyl-1H-1,2,3-triazole derivatives of carbohydrates. Eur. J. Med. Chem. 2009, 44, 373-383.

29. Boddy, I.K.; Briggs, G.G.; Harrison, R.P.; Jones, T.H.; Omahony, M.J.; Marlow, I.D.; Roberts, B.G.; Willis, R.J.; Bardsley, R.; Reid, J. The synthesis and insecticidal activity of a series of 2-aryl-1,2,3triazoles. Pestic. Sci. 1996, 48, 189-196.

30. Yan, M.; Shi, D.Q.; Xiao, L.X. Synthesis and herbicidal activity of novel $N$-(4,6-disubstituted pyrimidin-2-yl)-1-substituted pyridyl(thiazolyl)methyl -5methyl-1H-1,2,3-triazole-4-carboxamides. Chin. J. Org. Chem. 2008, 28, 1012-1015. 
31. Jordao, A.K.; Ferreira, V.F.; Souza, T.M.L.; Faria, G.G.D.; Machado, V.; Abrantes, J.L.; de Souza, M.; Cunha, A.C. Synthesis and anti-HSV-1 activity of new 1,2,3-triazole derivatives. Bioorg. Med. Chem. 2011, 19, 1860-1865.

32. Zou, Y.; Zhao, Q.J.; Hu, H.G.; Hu, L.N.; Yu, S.C.; Xu, M.J.; Wu, Q.Y. Synthesis and in vitro antitumor activities of xanthone derivatives containing 1,4-disubstituted-1,2,3-triazole moiety. Arch. Pharm. Res. 2012, 35, 2093-2104.

33. Patpi, S.R.; Pulipati, L.; Yogeeswari, P.; Sriram, D.; Jain, N.; Sridhar, B.; Murthy, R.; Devi, T.A.; Kalivendi, S.V.; Kantevari, S. Design, synthesis, and structure-activity correlations of novel dibenzo b,d furan, dibenzo b,d thiophene, and N-methylcarbazole clubbed 1,2,3-triazoles as potent inhibitors of Mycobacterium tuberculosis. J. Med. Chem. 2012, 55, 3911-3922.

34. Bakunov, S.A.; Bakunova, S.M.; Wenzler, T.; Ghebru, M.; Werbovetz, K.A.; Brun, R.; Tidwell, R.R. Synthesis and antiprotozoal activity of cationic 1,4-diphenyl-1H-1,2,3-triazoles. J. Med. Chem. 2009, 53, 254-272.

35. D’Hooghe, M.; Vandekerckhove, S.; Mollet, K.; Vervisch, K.; Dekeukeleire, S.; Lehoucq, L.; Lategan, C.; Smith, P.J.; Chibale, K.; De Kimpe, N. Synthesis of 2-amino-3-arylpropan-1-ols and 1-(2,3-diaminopropyl)-1,2,3-triazoles and evaluation of their antimalarial activity. Beilstein J. Org. Chem. 2011, 7, 1745-1752.

36. Saikia, B.; Saikia, P.P.; Goswami, A.; Barua, N.C.; Saxena, A.K.; Suri, N. Synthesis of a novel series of 1,2,3-triazole-containing artemisinin dimers with potent anticancer activity involving Huisgen 1,3-dipolar cycloaddition reaction. Synthesis 2011, 19, 3173-3179.

37. Su, N.N.; Xiong, L.X.; Yu, S.J.; Zhang, X.; Cui, C.; Li, Z.M.; Zhao, W.G. Larvicidal activity and click synthesis of 2-alkoxyl-2-(1,2,3-triazole-1-yl)acetamide library. Comb. Chem. High T. Scr. 2013, 16, 484-493.

38. Aher, N.G.; Pore, V.S.; Mishra, N.N.; Kumar, A.; Shukla, P.K.; Sharma, A.; Bhat, M.K. Synthesis and antifungal activity of 1,2,3-triazole containing fluconazole analogues. Bioorg. Med. Chem. Lett. 2009, 19, 759-763.

39. Pore, V.S.; Jagtap, M.A.; Agalave, S.G.; Pandey, A.K.; Siddiqi, M.I.; Kumar, V.; Shukla, P.K. Synthesis and antifungal activity of 1,5-disubstituted-1,2,3-triazole containing fluconazole analogues. Med. Chem. Commun. 2012, 3, 484-488.

40. Phillips, O.A.; Udo, E.E.; Abdel-Hamid, M.E.; Varghese, R. Synthesis and antibacterial activity of novel 5-(4-methyl-1H-1,2,3-triazole) methyl oxazolidinones. Eur. J. Med. Chem. 2009, 44, 3217-3227.

41. Behbehani, H.; Ibrahim, H.M.; Makhseed, S.; Mahmoud, H. Applications of 2-arylhydrazononitriles in synthesis: Preparation of new indole containing 1,2,3-triazole, pyrazole and pyrazolo 1,5-a pyrimidine derivatives and evaluation of their antimicrobial activities. Eur. J. Med. Chem. 2011, 46, 1813-1820.

42. Lofroth, J.E.; Ungell, A.L. Antithrombotic formulation, process for its manufacturing, and use thereof. US Patent: 5795896 A, 1998.

43. Kolb, H.C.; Finn, M.G.; Sharpless, K.B. Click chemistry: Diverse chemical function from a few good reactions. Angew. Chem. Int. Edit. 2001, 40, 2004-2021.

44. Roger, R.; Neilson, D.G. The chemistry of imidates. Chem. Rev. 1961, 61, 179-211. 
45. Carey, F.A.; Sundberg, R.J. Advanced Organic Chemistry, 5th ed.; Springer: Charlottesville, VA, USA, 2007; pp. 782-784.

46. Pinner, A.; Klein, F. Umwandlung der Nitrile in Imide. Ber. Dtsch. Chem. Ges. 1878, 11, 1475-1487.

47. Costa, M.S.; Boechat, N.; Rangel, E.A.; da Silva, F.D.; de Souza, A.M.T.; Rodrigues, C.R.; Castro, H.C.; Junior, I.N.; Lourenco, M.C.S.; Wardell, S.; et al. Synthesis, tuberculosis inhibitory activity, and SAR study of $N$-substituted-phenyl-1,2,3-triazole derivatives. Bioorg. Med. Chem. 2006, 14, 8644-8653.

48. Wang, X.L.; Wan, K.; Zhou, C.H. Synthesis of novel sulfanilamide-derived 1,2,3-triazoles and their evaluation for antibacterial and antifungal activities. Eur. J. Med. Chem. 2010, 45, 4631-4639.

49. Ozden, S.; Atabey, D.; Yildiz, S.; Goker, H. Synthesis and potent antimicrobial activity of some novel methyl or ethyl ${ }^{1}$ H-benzimidazole-5-carboxylates derivatives carrying amide or amidine groups. Bioorg. Med. Chem. 2005, 13, 1587-1597.

50. Tahghighi, A.; Marznaki, F.R.; Kobarfard, F.; Dastmalchi, S.; Mojarrad, J.S.; Razmi, S.; Ardestani, S.K.; Emami, S.; Shafiee, A.; Foroumadi, A. Synthesis and antileishmanial activity of novel 5-(5-nitrofuran-2-y1)-1,3, 4-thiadiazoles with piperazinyl-linked benzamidine substituents. Eur. J. Med. Chem. 2011, 46, 2602-2608.

51. Makhsumov, A.G.; Rakhimova, I.V.; Sladkov, A.M. Synthesis of propargyl ethers, possessing bactericidal properties. Russ. Chem. Bull. 1968, 17, 2450-2452.

52. Chen, N.C. Bioassay of Pesticides; Beijing Agricultural University Press: Beijing, China, 1991; pp. 161-162.

53. Li, S.Z.; Zhang, S.H.; Liu, S.F. Simple bioassay method of new Fungicide against Botrytis cinerea-cucumber cotyledon method. Acta Agric. Boreal. Sin. 1991, 6, 94-99.

Sample Availability: Samples of the compounds 2, 3, 7, 8, 4a-c, 9a-b, 12, 14, 15a-b, 16a-b, 17a-b, 18, 19 are available from the authors.

(C) 2014 by the authors; licensee MDPI, Basel, Switzerland. This article is an open access article distributed under the terms and conditions of the Creative Commons Attribution license (http://creativecommons.org/licenses/by/3.0/). 